\title{
The MAT Locus Genes Play Different Roles in Sexual Reproduction and Pathogenesis in Fusarium graminearum
}

\author{
Qian Zheng ${ }^{19}$, Rui Hou ${ }^{1,29}$, Juanyu, Zhang ${ }^{1}$, Jiwen Ma ${ }^{1}$, Zhongshou Wu' ${ }^{1}$ Guanghui Wang ${ }^{1,2}$, \\ Chenfang Wang ${ }^{1}$, Jin-Rong $\mathrm{Xu}^{2 *}$
}

1 State Key Laboratory of Crop Stress Biology for Arid Areas, College of Plant Protection, Northwest Agricultural and Forestry University, Yangling, Shaanxi, China, 2 Department of Botany and Plant Pathology, Purdue University, West Lafayette, Indiana, United States of America

\begin{abstract}
Sexual reproduction plays a critical role in the infection cycle of Fusarium graminearum because ascospores are the primary inoculum. As a homothallic ascomycete, F. graminearum contains both the MAT1-1 and MAT1-2-1 loci in the genome. To better understand their functions and regulations in sexual reproduction and pathogenesis, in this study we assayed the expression, interactions, and mutant phenotypes of individual MAT locus genes. Whereas the expression of MAT1-1-1 and MAT12-1 rapidly increased after perithecial induction and began to decline after 1 day post-perithecial induction (dpi), the expression of MAT1-1-2 and MAT1-1-3 peaked at 4 dpi. MAT1-1-2 and MAT1-1-3 had a similar expression profile and likely are controlled by a bidirectional promoter. Although none of the MAT locus genes were essential for perithecium formation, all of them were required for ascosporogenesis in self-crosses. In outcrosses, the mat11-1-2 and mat11-1-3 mutants were fertile but the mat1-1-1 and mat1-2-1 mutants displayed male- and female-specific defects, respectively. The mat1-2-1 mutant was reduced in FgSO expression and hyphal fusion. Mat1-1-2 interacted with all other MAT locus transcription factors, suggesting that they may form a protein complex during sexual reproduction. Mat1-1-1 also interacted with FgMcm1, which may play a role in controlling cell identity and sexual development. Interestingly, the mat 1-1-1 and mat 1-2-1 mutants were reduced in virulence in corn stalk rot assays although none of the MAT locus genes was important for wheat infection. The MAT1-1-1 and MAT1-2-1 genes may play a host-specific role in colonization of corn stalks.
\end{abstract}

Citation: Zheng Q, Hou R, Juanyu, Zhang, Ma J, et al. (2013) The MAT Locus Genes Play Different Roles in Sexual Reproduction and Pathogenesis in Fusarium graminearum. PLoS ONE 8(6): e66980. doi:10.1371/journal.pone.0066980

Editor: Jae-Hyuk Yu, University of Wisconsin - Madison, United States of America

Received March 2, 2013; Accepted May 13, 2013; Published June 24, 2013

Copyright: (c) 2013 Zheng et al. This is an open-access article distributed under the terms of the Creative Commons Attribution License, which permits unrestricted use, distribution, and reproduction in any medium, provided the original author and source are credited.

Funding: This work was supported the National Major Project of Breeding for New Transgenic Organisms (2012ZX08009003), the National Basic Research Program of China (2012CB114002; 2013CB127703), and a grant from the USDA-NIFA (2010 65110 20439). The funders had no role in study design, data collection and analysis, decision to publish, or preparation of the manuscript.

Competing Interests: The authors have declared that no competing interests exist.

*E-mail: jinrong@purdue.edu

9 These authors contributed equally to this work.

\section{Introduction}

Fusarium graminearum (teleomorph Gibberella zeae) is a causal agent of Fusarium head blight (FHB) or scab of wheat, barley, and other small grains worldwide $[1,2]$. This fungal pathogen overwinters on plant debris and produces ascospores in the spring to initiate infection of flowering tissues of wheat and other host plants. Unlike most pathogenic fungi, sexual reproduction plays a critical role in the infection cycle of $F$. graminearum because ascospores are the primary inoculum [3]. Flowering wheat heads are susceptible to Fusarium infection from anthesis through the milk stages. Under favorable environmental conditions, FHB can cause severe yield losses. In addition, $F$. graminearum is a producer of deoxynivalenol $(\mathrm{DON})$ and zearalenone. Infested grains are often contaminated with these harmful mycotoxins [4].

As a homothallic ascomycete, F. graminearum has both MAT1-1 and MAT1-2-1 that are arranged in tandem [5]. Similar to mutants deleted of the entire mating locus, the mat1-1 and mat1-21 deletion mutants were sterile in self-crosses [6]. Similar to many other Sordariomycetes, MAT1-1 of F. graminearum contains three putative transcription factor (TF) genes known as MAT1-1-1, MAT1-1-2, and MAT1-1-3 [5]. Mat1-1-1 is the well-conserved alphal domain protein but the putative DNA-binding domain of Mat1-1-2 or Mat1-1-3 (HMG-like) has not been functionally characterized. In contrast, MAT1-2-1 contains a single transcription factor gene that encodes a HMG-box protein. Recently, it has been shown that alpha domain proteins and HMG proteins encoded by fungal mating type genes are evolutionally related [7]. The MAT1-2-3 gene adjacent to MAT1-2-1 was predicted to be a new MAT gene [8]. However, unlike other MAT TF genes, MAT12-3 has no DNA-binding motif. Deletion of MAT1-2-3 had no effects on sexual reproduction [9].

The functions of MAT locus genes have been characterized in several Sordariomycetes, including Neurospora crassa, Podospora anserina, and Sordaria macrospora [10,11]. In general, the MAT TFs are dispensable for vegetative growth and asexual reproduction. In most filamentous ascomycetes, the MAT1-1-1 and MAT1-2-1 orthologs are essential for mating and mating type specificity. In $\mathcal{N}$. crassa, the matA-1 and mata- 1 mutants were sterile but the mat $A-2$ and matA-3 mutants were only slightly reduced in fertility [12]. 
Even the matA-2 matA-3 double mutant was still fertile although it was significantly reduced in perithecium and ascospore formation, suggesting that these genes may have overlapping functions in sexual development. In $S$. macrospora, a homothallic ascomycete closely related to $\mathcal{N}$. crassa, the $S m t A-1$ (MAT1-1-1) and SmtA-3 (MAT1-1-3) deletion mutants produced the same number of perithecia and mature, viable ascospores as the wild type. In contrast, mature perithecia were not observed in the SmtA-2 (MAT1-1-2) deletion mutant, which was blocked in sexual development at the stage of early protoperithecium formation [10].

In Saccharomyces cerevisiae, only the MAT locus genes in the MAT locus are transcribed. The MAT genes in the $H M L$ and $H M R$ loci are silenced [13,14]. The silenced HML $\alpha$ or HMLa loci function as the donor sequences for gene conversion during mating type switching. In MAT $\alpha$ cells, Mat $\alpha 1$ interacts with $\mathrm{Mcml}$ to express $\alpha$-specific genes and MAT $\alpha 2$ inhibits the $a$-specific genes by binding with $\mathrm{Mcm} 1 / \mathrm{Tup} 1 / \mathrm{Ssn} 6$. In diploid cells, Matal interacts with Mat $\alpha 2$ to inhibit haploid-specific genes, such as $a$ - or $\alpha$-specific pheromone and pheromone receptor genes [15,16,17]. Mating type switching has not been observed in filamentous fungi although some homothallic fungi contain both MAT idiomorphs in the genome. In $\mathcal{N}$. crassa, the interaction between mat $\mathrm{A}-1$ and mata-1 proteins was detected [18]. The matA-2 and matA-3 proteins weakly interact with each other in yeast two-hybrid assays [19]. In S. macrospora, SmtA-1 is known to interact with Mcml and Smta-1 but not with SmtA-2 or SmtA-3 [20,21]. In the heterothallic ascomycete $P$. anserina, no direct interactions among $M A T$ locus TF proteins encoded by the opposite mating type loci were detected in yeast two-hybrid assays. Nevertheless, the mat- $A$ specific transcription factors FMR1 and $S M R 2$ that are orthologous to matA-1 and matA-3 of $\mathcal{N}$. crassa interacted with each other [22].

The MAT locus genes are well conserved in $F$. graminearum. Because proper regulation of the expression, activation, and interaction of MAT locus genes is important for growth and differentiation in a homothallic fungus, in this study we functionally characterized individual MAT locus genes. All four MAT locus genes in F. graminearum had induced expression during sexual reproduction and were required for ascosporogenesis. However, none of them was essential for growth and infection of flowering wheat heads, which is similar to a recent report on functional characterization of MAT locus genes [9] that was published during the preparation of this manuscript. Although we failed to detect the direct interaction between Mat1-1-1 and Mat1-2, Mat1-1-2 interacts with all other MAT locus genes in yeast two hybrid-assays, suggesting that they may form protein complexes during sexual reproduction. Both MAT1-1-1 and MAT1-2-1 were rapidly induced during early stages of sexual development then gradually declined. The expression of MAT1-1-2 and MAT1-1-3 peaked at 4 days post-perithecial induction, which was later than the up-regulation of MAT1-1-1 and MAT1-2-1. MAT1-1-2 and MAT1-1-3 had similar expression profiles and they may have overlapping functions during late stages of perithecium development. In addition to the male- and femalespecific defects observed in the mat1-1-1 and mat1-2-1 deletion mutants, respectively, we found that MAT1-1-1 and MAT1-2-1 are important for colonization of corn stalks, suggesting that they play a host-specific role in pathogenesis.

\section{Results}

\section{Differential Expression of MAT Locus Genes in F. graminearum}

In the closely clinked MAT idiomorphs, the MAT1-2-1 (FGSG_008993) and MAT1-1-1 (FGSG_008992) genes share a 139-bp terminator region that contains a CTGTACAG palindromic sequence. In contrast, MAT1-1-2 (FGSG_008991; 259983-261563-) and MAT1-1-3 (FGSG_008990; 262020262759+) have the head-to-head arrangement and are only 457bp apart (Fig. 1A), suggesting that they share a bidirectional promoter [23]. In comparison with the corresponding ESTs, the putative transcription initiation sites of these two genes are at 261816 and 262075, respectively. The 259-bp sequence between the transcription initiation sites of MAT1-1-2 and MAT1-1-3 contains a palindromic GAAAGCTTTC sequence, which consists of two CTTTC sequences in different strands. In $\mathcal{N}$. crassa, the mata-1 binding site is CTTTG [24].

To determine the expression profiles of MAT locus genes during sexual reproduction, we isolated RNA from aerial hyphae scrapped off 7-day-old carrot agar plates and fungal biomasses harvested from carrot agar cultures 1, 2, 4, 6, and 10 days postperithecial induction (dpi). In aerial hyphae harvested before perithecial induction, the expression of MAT locus genes was not detectable or relatively low (Fig. 1B), suggesting that their expression was suppressed during vegetative growth. The expression of MAT1-1-1 and MAT1-2-1 increased approximately 11and 10-fold, respectively at $1 \mathrm{dpi}$, and decreased after that (Fig. 1B). Therefore, these two MAT locus TF genes are likely important for earlier stages of sexual reproduction. For the MAT1-1-2 and MAT1-1-3 genes, their expression also increased after perithecial induction but peaked at 4 dpi (Fig. 1B). A similar expression profile of MAT1-1-2 and MAT1-1-3 is consistent with the fact that they share a common promoter (Fig. 1). These two genes may play more important roles in later stages than in earlier stages of perithecium development. When compared with the expression levels of other MAT TF genes, MAT1-2-1 had the highest level at 0, 1, 4, and 7 dpi (Fig. 1C). MAT1-1-1 had a higher expression level than MAT1-1-2 and MAT1-1-3 at 0, 1, 4, and 10 dpi (Fig. 1C). Similar trends were observed in an earlier study [9].

\section{Expression and Subcellular Localization of MAT Locus Genes}

To determine their expression and subcellular localization during sexual development, we generated the knock-in GFP fusion transformants for the four MAT locus TF genes of $F$. graminearum (Fig. S1). All the resulting GFP knock-in transformants (Table 1) were normal in vegetative growth and sexual reproduction, indicating that GFP insertion had no obvious effects on their functions. However, GFP signals were very weak (Fig. 2A) or not detectable (Fig. S1) in vegetative hyphae or ascogenous tissues in all these knock-in transformants, including the MAT1-1-3-GFP strain (Fig. 2A). Weak GFP signals were too faint to be distinguished from the auto-fluorescence of perithecium tissues and we failed to detect the localization of GFP signals in the nucleus in any of these GFP knock-in transformants.

In addition to the knock-in transformants, we also generated transformants expressing the $\mathrm{P}_{\text {TrpC }}-M A T 1-1-3-\mathrm{GFP}$ and $\mathrm{P}_{\text {TrpC }}-$ MAT1-2-1-GFP fusion constructs ectopically. In the $\mathrm{P}_{\mathrm{TrpC}}-M A T 1-$ 1-3-GFP transformant, GFP signals in the cytoplasm were still relatively weak but appeared to be stronger than the knock-in transformant (Fig. 2B). Localization of GFP-signals to the nucleus was observed in vegetative hyphae of the MAT1-2-1-GFP transformant (Fig. 2B). Nevertheless, we still failed to observe GFP signals that were stronger than the fluorescent background in perithecium tissues of the $\mathrm{P}_{\text {TrpC }}-M A T 1-1-3-G F P$ and $\mathrm{P}_{\text {TrpC- }}$ MAT1-2-1-GFP transformants. It is possible that the GFP fusion proteins of these MAT locus gene are not stable or degraded rapidly during sexual reproduction. 
A

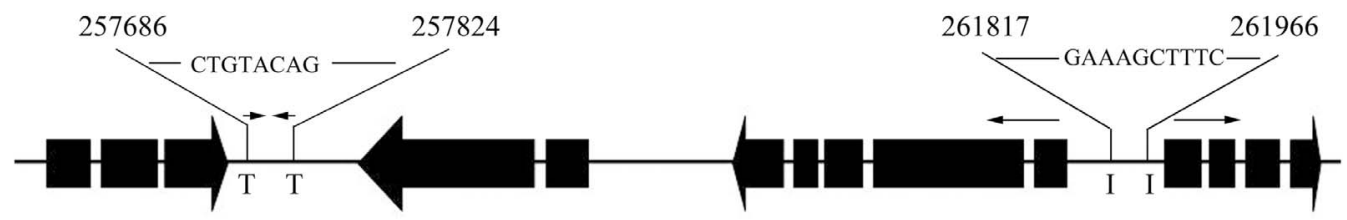
MATI-2-1
MAT1-1-1
MATI-1-2
MATI-1-3

B

$\square 0 \mathrm{~d}$

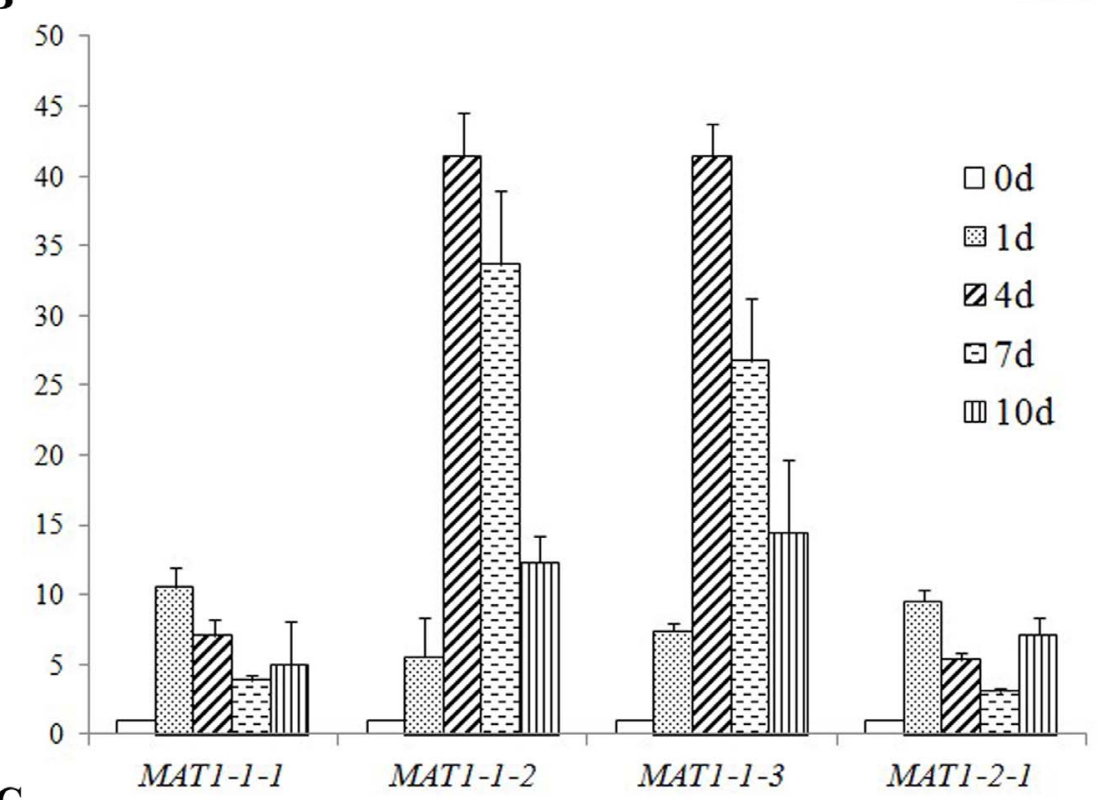

C

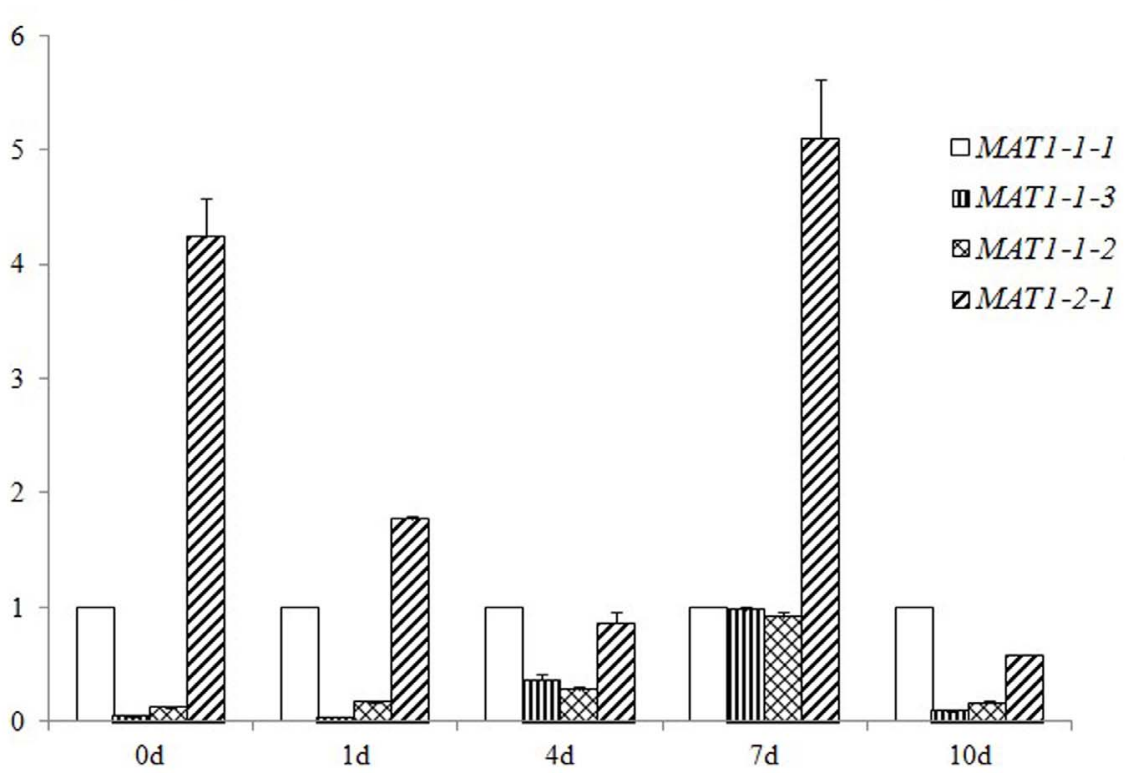

Figure 1. Chromosomal positions and expression levels of MAT locus genes. A. Diagram of four MAT locus genes. The thick black bars and arrows represent the exons and directions of MAT1-2-1, MAT1-1-1, MAT1-1-2, and MAT1-1-3 genes. I and T represent the initiation and termination sites of transcription. CTGTACAG and GAAAGCTTTC are palindromic sequences. B. Relative expression levels of individual MAT locus genes at 1, 2, 4, 6 , and 10 days post-perithecial induction (dpi) were assayed by qRT-PCR. The expression level of individual genes in aerial hyphae collected from 7day-old carrot agar cultures before perithecial induction (day 0 control) was arbitrarily set to 1 . C. Relative expression levels of four MAT locus TF genes at 1, 2, 4, 6, and $10 \mathrm{dpi}$. For each time point, the expression level of MAT1-1-1 was set to 1 . Mean and standard error were calculated from three independent biological replicates. doi:10.1371/journal.pone.0066980.g001 
A $\quad$ G90
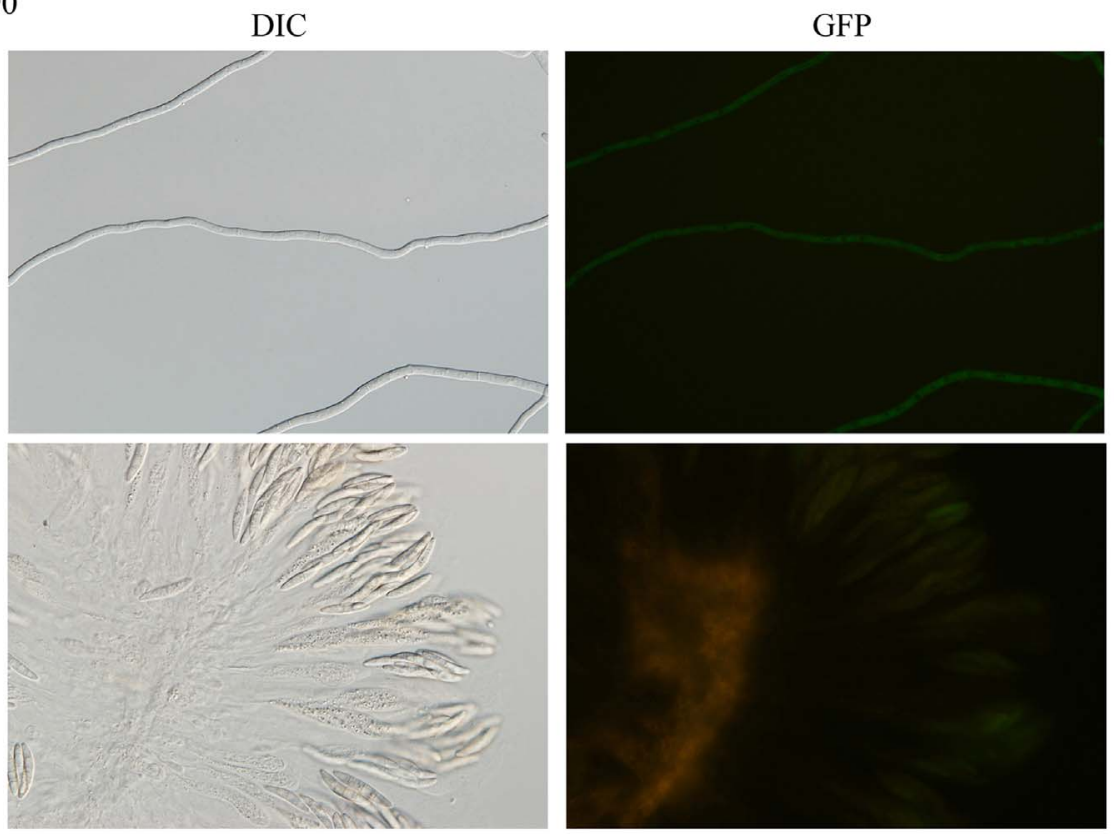

B

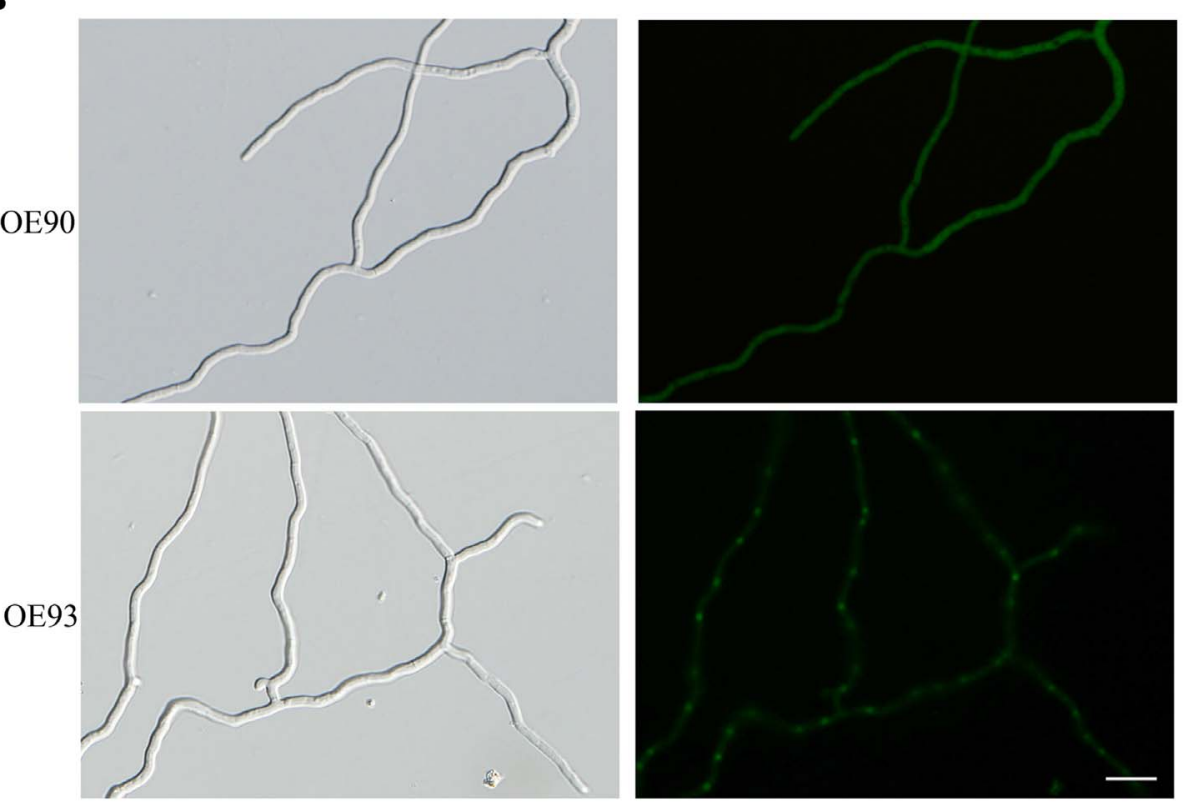

Figure 2. Expression and subcellular localization of $M A T$ locus TF genes. A. Weak GFP signals in vegetative hyphae (upper panels) and asci (lower panels) of the MAT1-1-3-GFP knock-in transformant. B. GFP signals in vegetative hyphae of the $\mathrm{P}_{\mathrm{Trpc}}-$ MAT1-1-3-GFP (90R) and $\mathrm{P}_{\text {Trpc }}-$ MAT1-2-1GFP (93R) transformants. The same field was observed by DIC or epifluorescence microscopy. Bar $=20 \mu \mathrm{m}$. doi:10.1371/journal.pone.0066980.g002

\section{Mutants Deleted of Individual MAT Locus Genes are Defective in Sexual Reproduction}

To determine the functions of MAT TFs, we generated gene replacement mutants deleted of individual MAT locus genes in the wild-type strain PH-1 (Fig. S2). For each gene, at least three independent knockout mutants were isolated (Fig. S2; Table 1) and found to have the same phenotypes described below (data not shown). None of the resulting mutants (Table 1) had obvious defects in the growth rate or colony morphology (Fig. S3). Conidiation and conidium morphology also were normal in these mutants (Fig. S4). Nevertheless, all the MAT locus gene deletion mutants were defective in sexual reproduction in self-crosses. At 7 days post-perithecial induction, all the mutants produced darkpigmented perithecia that were smaller than the wild-type perithecia (Fig. 3A). These mutant perithecia were sterile and failed to produce ascospore cirrhi. When cracked open, asci or ascospores were not observed in perithecia formed by the mutants (Fig. 3B).

To further characterize the defects of MAT locus TF deletion mutants in sexual reproduction, thick-sections of the wild-type and mutant perithecia were examined under a light microscope (Fig. 3C). In contrast to ascogenous hyphae and asci at the base 
Table 1. Strains used in this study.

\begin{tabular}{|c|c|c|}
\hline Strains & Genotype & References \\
\hline $\mathrm{PH}-1$ & Wild type & [53] \\
\hline mat1-1 & mat1-1 deletion mutant of $\mathrm{PH}-1$ & {$[6]$} \\
\hline M92-2 & mat1-1-1 deletion mutant of $\mathrm{PH}-1$ & This study \\
\hline M92-5 & mat1-1-1 deletion mutant of $\mathrm{PH}-1$ & This study \\
\hline M92-15 & mat1-1-1 deletion mutant of $\mathrm{PH}-1$ & This study \\
\hline M91-3 & mat1-1-2 deletion mutant of $\mathrm{PH}-1$ & This study \\
\hline M91-8 & mat1-1-2 deletion mutant of $\mathrm{PH}-1$ & This study \\
\hline M91-21 & mat1-1-2 deletion mutant of $\mathrm{PH}-1$ & This study \\
\hline M90-1 & mat1-1-3 deletion mutant of $\mathrm{PH}-1$ & This study \\
\hline M90-11 & mat1-1-3 deletion mutant of $\mathrm{PH}-1$ & This study \\
\hline M90-12 & mat1-1-3 deletion mutant of $\mathrm{PH}-1$ & This study \\
\hline M90-20 & mat1-1-3 deletion mutant of $\mathrm{PH}-1$ & This study \\
\hline M93-1 & mat1-2-1 deletion mutant of $\mathrm{PH}-1$ & This study \\
\hline M93-15 & mat1-2-1 deletion mutant of $\mathrm{PH}-1$ & This study \\
\hline M93-7 & mat1-2-1 deletion mutant of $\mathrm{PH}-1$ & This study \\
\hline S6 & Fgso deletion mutant of $\mathrm{PH}-1$ & This study \\
\hline G92-9 & MAT1-1-1-GFP knock-in transformant & This study \\
\hline G91-11 & MAT1-1-2-GFP knock-in transformant & This study \\
\hline G91-21 & MAT1-1-2-GFP knock-in transformant & This study \\
\hline G90 & MAT1-1-3-GFP knock-in transformant & This study \\
\hline G93 & MAT1-2-1-GFP knock-in transformant & This study \\
\hline B7-1 & MAT1-1-1-YFPN+MAT1-1-2-YFPC transformant & This study \\
\hline B7-2 & MAT1-1-1-YFPN+MAT1-1-2-YFPC transformant & This study \\
\hline B8-1 & MAT1-2-1-YFPN+MAT1-1-2-YFPC transformant & This study \\
\hline B8-2 & MAT1-2-1-YFPN+MAT1-1-2-YFPC transformant & This study \\
\hline B9-1 & MAT1-1-3-YFPN+MAT1-1-2-YFPC transformant & This study \\
\hline B9-2 & MAT1-1-3-YFPN+MAT1-1-2-YFPCtransformant & This study \\
\hline B10-1 & MAT1-1-3-YFPC+MAT1-2-1-YFPC transformant & This study \\
\hline B10-2 & MAT1-1-3-YFPN+MAT1-2-1-YFPCtransformant & This study \\
\hline OE90 & $\mathrm{P}_{\text {TrpC }}-M A T 1-1-3-G F P$ transformant of $\mathrm{PH}-1$ & This study \\
\hline OE93 & $\mathrm{P}_{\text {TrpC }}-M A T 1-2-1-G F P$ transformant of $\mathrm{PH}-1$ & This study \\
\hline Comp90 & $\begin{array}{l}\text { mat1-1-3/MAT1-1-3-GFP complemented } \\
\text { transformant }\end{array}$ & This study \\
\hline Comp91 & $\begin{array}{l}\text { mat1-1-2/MAT1-1-2-GFP complemented } \\
\text { transformant }\end{array}$ & This study \\
\hline
\end{tabular}

of the wild-type perithecia, perithecia formed by the MAT locus gene deletion mutants appeared to be blocked in the development of ascogenous hyphae and tended to have thicker layers of perithecium wall (Fig. 3C). No asci or ascospores were observed. These results indicate that none of the MAT locus genes is essential for the initiation of perithecium development. However, the development of asci or ascogenous hyphae and enlargement of young perithecia require proper functions of all four MAT locus genes in self-crosses.

\section{The mat1-1-1 and mat1-2-1 Deletion Mutants are Defective in Infection of Corn Stalks}

We also assayed DON production and virulence on flowering wheat heads with the MAT locus TF gene deletion mutants. In comparison with the wild type, none of these mutants had obvious changes in virulence in infection assays with flowering wheat heads, which was consistent with a previous report [9]. However, in infection assays with corn stalks, we noticed that the mat1-1-1 and mat1-2-1 deletion mutants, but not the mat1-1-2 and mat1-1-3 mutants, were reduced in virulence (Fig. 4). When the stalk rot areas were measured, the virulence of the mat1-1-1 and mat1-2-1 deletion mutants were reduced $66 \%$ and $77 \%$ in comparison with that of the wild type. In nature, corn also is a host plant for $F$. graminearum, which often produces abundant perithecia on corn stalks. The defects of the mat1-1-1 and mat1-2-1 deletion mutants in corn stalk rot assays suggest that the mating type locus may play a host-specific role in plant infection.

\section{Mat1-1-2 Interacts with All other MAT Locus TF Genes in Yeast Two-hybrid Assays}

Because of similar defects of the MAT TF mutants in sexual reproduction, it is likely that these $M A T$ locus genes interact with each other and function in a protein complex (similar to the Mat $a$ Mat $\alpha$ interaction in $S$. cerevisiae) during sexual reproduction or ascosporogenesis inside perithecia. To test this hypothesis, we amplified the cDNA clones of all the MAT locus genes and assayed for their pair-wise interactions. In yeast two-hybrid assays, Matl1-1 interacted with Mat1-1-2 but not with Mat1-2 or Mat1-1-3. Mat1-1-2 and Mat1-1-3 interacted with each other and both of them interacted with Mat1-2 (Fig. 5). Although it is surprising that the direct interaction between Mat1-1-1 and Mat1-2 was not observed, Mat1-1-2 interacted with all the other MAT locus TF proteins, which may bring Mat1-1-1 and Mat1-2 together in a protein complex in $F$. graminearum.

In attempt to further characterize the interactions among the MAT locus genes, we generated the $\mathrm{BiFC}$ constructs and transformed them in pairs into $\mathrm{PH}-1$. In the resulting transformants (Table 1), we assayed for YFP signals in conidia, germlings, hyphae, and perithecium tissues. Unfortunately, we failed to observe obvious YFP signals in repeated experiments in all the resulting transformants, including the MAT1-1-2-YFPC MAT1-11-YFPN transformant (Table 1). In addition to the possibility that these MAT locus genes are expressed at a relatively low level, their YFP fusion proteins may be unstable or their interactions are too transient.

\section{Mat1-1-1 Interacts with FgMcm1}

In the budding yeast, Mcml interacts with Mata or Mat $\alpha$ to regulate different processes in haploid or diploid cells $[25,26]$. We also assayed the interaction of different MAT locus genes with the orthologs of Mcml in F. graminearum. In yeast two-hybrid assays, only Mat1-1-1 interacted with FgMcml (Fig. 6). Although Mat11-2 interacted with all other MAT locus genes, it did not interact with $\mathrm{FgMcml}$.

\section{MAT1-1-1 and MAT1-2-1 Play Different Roles in Outcrosses}

Although it is a homothallic fungus, out-crossing is possible in $F$. graminearum. In the perithecia formed by the mat1-1-2 (male) and mat1-1-3 (female) or the reciprocal crosses, normal perithecia and ascospore cirrhi were produced, indicating that these two mutants were not defective in male or female fertility and MAT1-1-2 mat11-3/mat1-1-2 MAT1-1-3 heterozygous dikaryotic or diploid cells were normal in the development of asci and ascospores.

In crosses with the mat1-1-2 and mat1-1-3 mutants, fertile perithecia were formed only when the mat1-1-1 or mat1-1 deletion mutant [6] was used as the female. When the mat1-1-1 mutant was used as the male, only sterile, small perithecia were formed, 
A PH-1 mat1-1-3

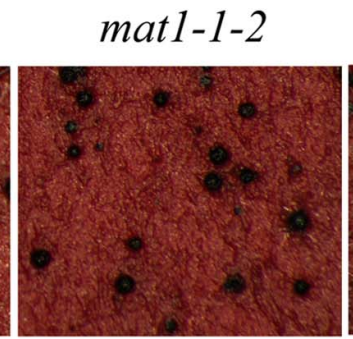

mat1-1-1

mat1-2-1
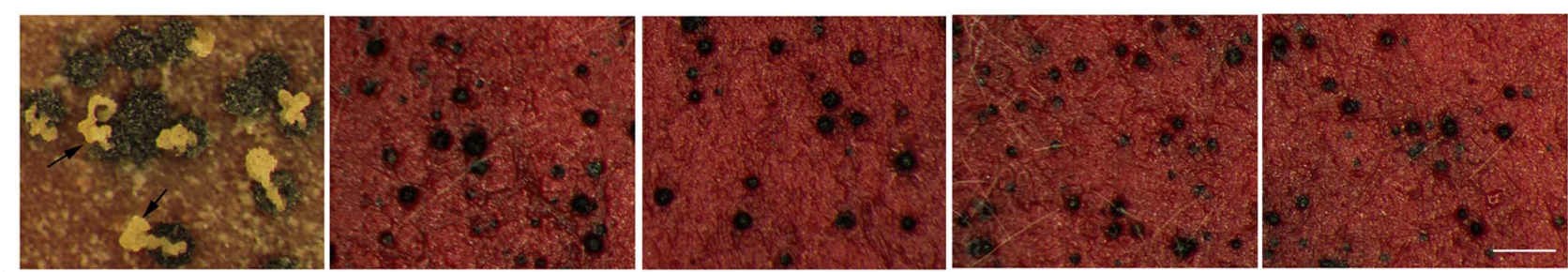

B
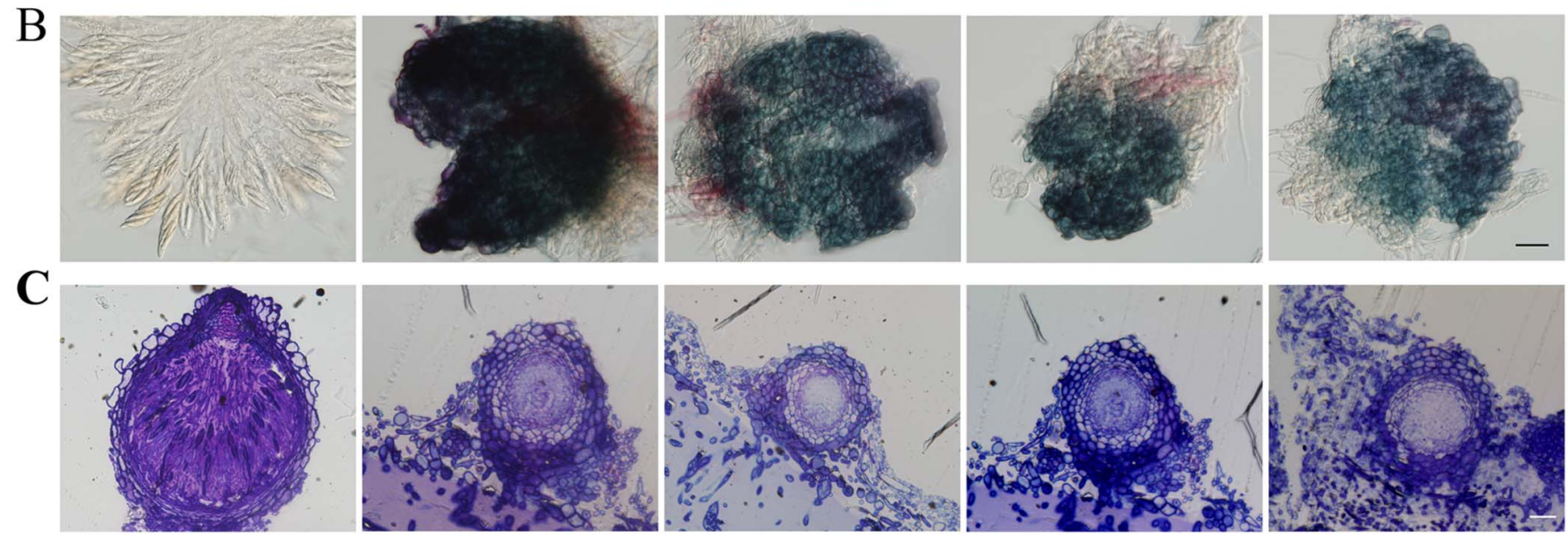

Figure 3. Defects of different MAT locus gene deletion mutants in self-crosses. A. Perithecia produced by 14-day-old carrot agar cultures of the wild type ( $\mathrm{PH}-1)$ and different MAT locus gene deletion mutants. Arrows pointed to the cirrhi. Bar $=1 \mathrm{~mm}$. B. Perithecia of PH-1 and the MAT locus gene deletion mutants were examined for ascus and ascospore development. Bar $=20 \mu \mathrm{m}$. C. Thick sections of representative perithecia produced by the wild type and mutant strains. Bar $=20 \mu \mathrm{m}$.

doi:10.1371/journal.pone.0066980.g003

suggesting that MAT1-1-1 is required for male fertility but dispensable for female fertility (Table 2; Fig. 6). In contrast, MAT1-2-1 may play a more important role in female fertility than in male fertility because the mat1-2-1 deletion mutant displayed a female-specific defect in crosses with the mat1-1-2 and mat1-1-3 mutants (Table 2; Fig. 6). In the crosses with the mat1-1-2 and mat1-1-3 mutants, normal, fertile perithecia were formed when the

\section{PH-1 mat1-1-3 mat1-1-2 mat1-1-1 mat1-2-1}

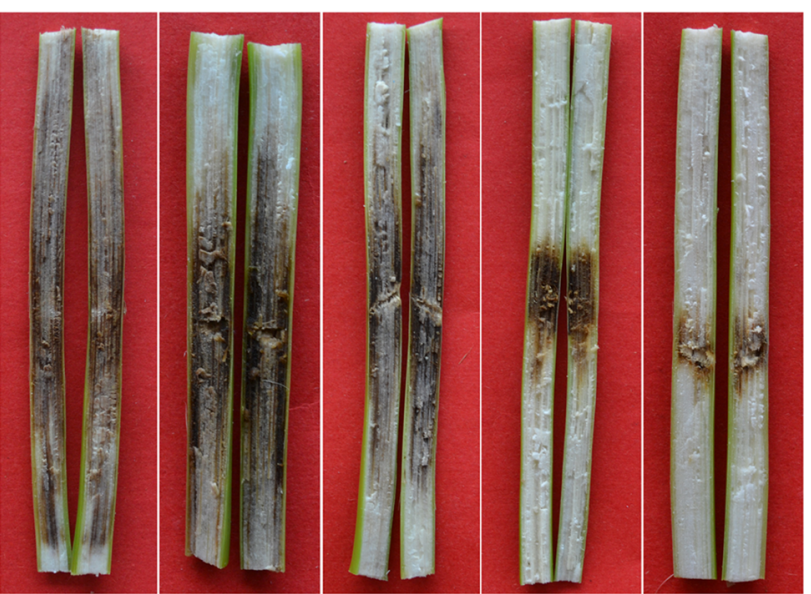

Figure 4.The mat1-1-1 and mat1-2-1 mutants were defective in corn stalk infection. Corn stalks inoculated with $\mathrm{PH}-1$ and four MAT locus gene deletion mutants. Stalk rot (discoloration) was restricted in plants inoculated with the mat1-1-1 and mat1-2-1 mutants. doi:10.1371/journal.pone.0066980.g004 mat1-2-1 mutant was used as the male. When the mat1-2-1 mutant was used as the female, most of the perithecia, similar to those of the mat1-2-1 mutant formed in self-crosses, were small and sterile. However, approximately $5 \%$ of them were similar to the wild-type perithecia in size and produced normal ascospores. These results indicate that MAT1-2-1 is not essential but important for female fertility in $F$. graminearum.

When the mat1-2-1 mutant was crossed as the female with the mat1-1-1 mutant, only smaller, sterile perithecia were formed (Table 2; Fig. 6). We failed to observe perithecia with ascospores in repeated experiments. These results were consistent with the defects of the mat1-2-1 and mat1-1-1 mutants in female and male fertility, respectively (Fig. 6). However, perithecia with ascospore cirrhi were produced by the mat1-1-1 (female) x mat1-2-1 (male) cross (Fig. 6). Therefore, the mat1-1-1 MAT1-2-1/MAT1-1-1 mat12-1 dikaryotic or diploid cells were normal in later stages of perithecium development and ascosporogenesis. These results further indicate that the MAT1-2-1 and MAT1-1-1 genes play critical roles in earlier stages of perithecium development.

\section{The FgSO Gene and Hyphal Fusion are Required for Sexual Reproduction}

Because the $S O$ gene is known to regulate hyphal fusion and female fertility in $\mathcal{N}$. crassa $[27,28]$, we generated the Fgso deletion mutant in $F$. graminearum by the split marker approach (Fig. S5). The Fgso mutant was sterile in self-crosses. When crosses with the mat1-1-1 and mat1-2-1 mutants, the Fgso mutant was female sterile but retained male fertility (Fig. 7A). Fertile perithecia and normal ascospores were observed only in crosses with the mat1-1-1 and mat1-2-1 mutants when the Fgso mutant functioned as the male. The Fgso1 (male) x mat1-2-1 (female) cross produced fewer fertile 
B
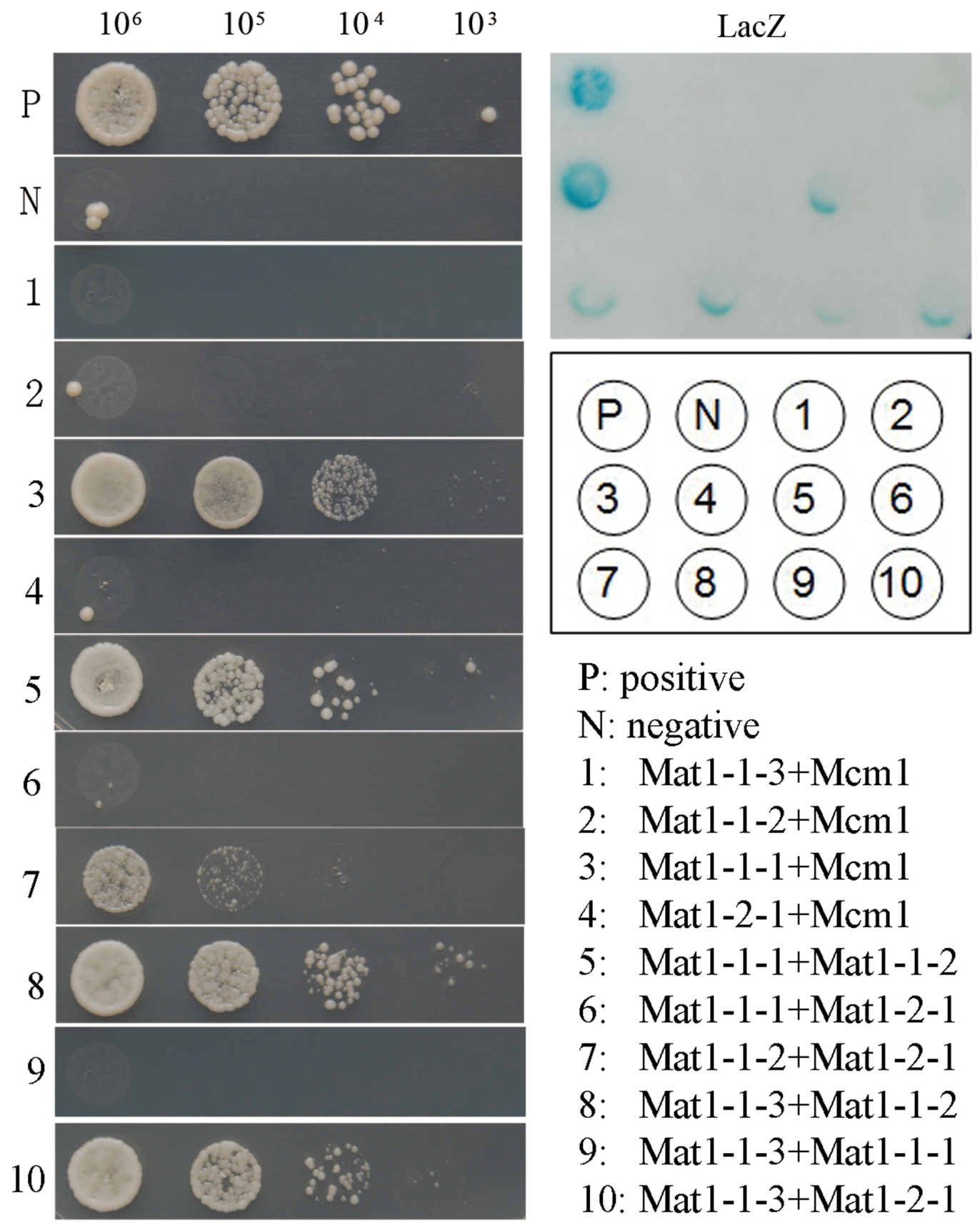

P: positive

$\mathrm{N}$ : negative

1: Mat1-1-3+Mcm 1

2: Mat1-1-2+Mcm 1

3: Mat1-1-1+Mcm 1

4: Mat1-2-1+Mcm 1

5: Mat1-1-1+Mat1-1-2

6: Mat1-1-1+Mat1-2-1

7: Mat1-1-2+Mat1-2-1

8: Mat1-1-3+Mat1-1-2

9: Mat1-1-3+Mat1-1-1

10: Mat1-1-3+Mat1-2-1

Figure 5. Yeast two-hybrid assays for the interactions among four MAT locus TFs. A. Different concentrations of yeast cells (cells/ml) of the transformants expressing the labeled bait and prey constructs were assayed for growth on SD-Leu-Trp-His plates. $\mathrm{P}$ and $\mathrm{N}$ were the positive and negative controls provided in the BD Matchmaker library construct kit. B. The same set of yeast transformants was assayed for $\beta$-galactosidase activities.

doi:10.1371/journal.pone.0066980.g005

perithecia than the Fgso1 (male) x mat1-1-1 (female) cross, which is consistent with the female-specific defects of the mat1-2-1 mutant.

Similar to the so mutant in $\mathcal{N}$. crassa, the Fgso deletion mutant was defective in hyphal fusion (Fig. 7B). In comparison with the wild type, hyphal fusion was normal in the mat1-1-1 mutant (Fig. 7B). In the mat1-2-1 deletion mutant, hyphal fusion still occurred but at a reduced frequency (Fig. 7B). When assayed by qRT-PCR, the expression level of $\mathrm{FgSO}$ was significantly reduced in the mat1-2-1 mutant but not in the mat1-1-1 mutant (Fig. 7C). Therefore, the defects of the mat1-2-1 mutant in hyphal fusion may be directly related to the down-regulation of FgSO expression. MAT1-2-1 may be involved in regulating the expression of FgSO during sexual reproduction in $F$. graminearum. 
A (mat1-2-1)
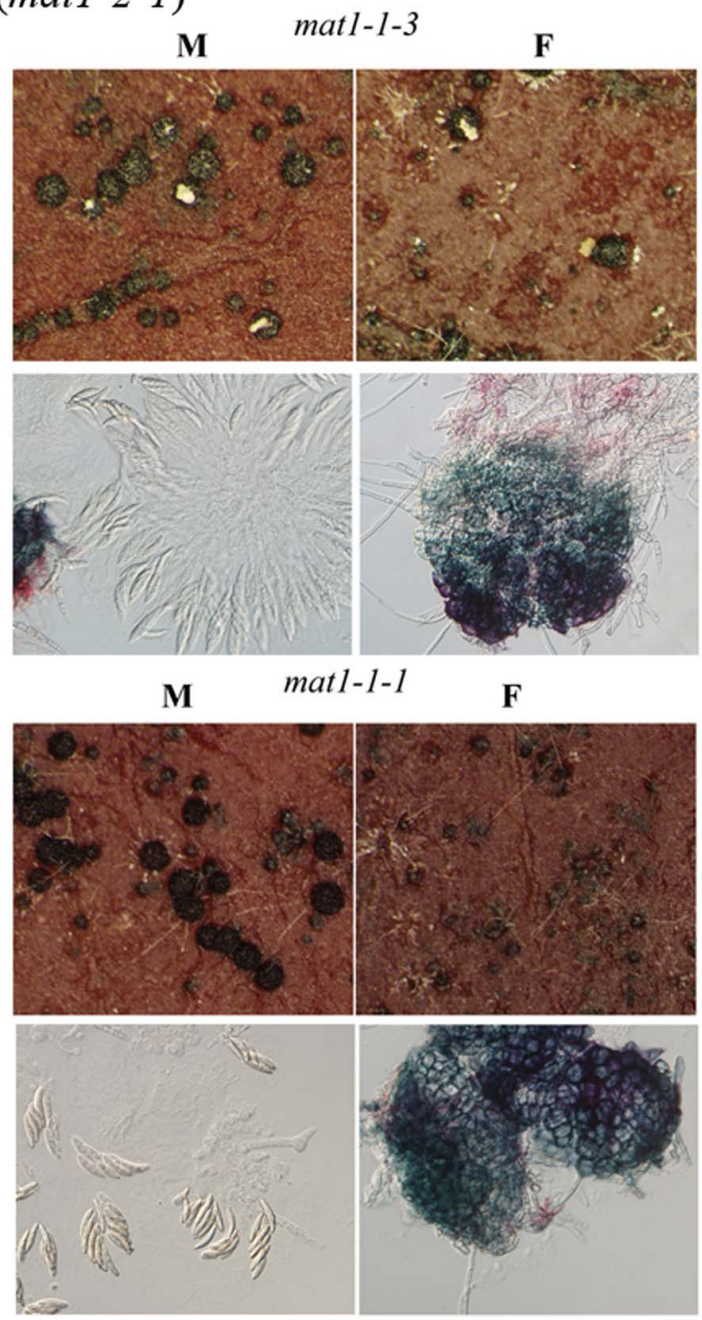

B (mat 1-1-1)
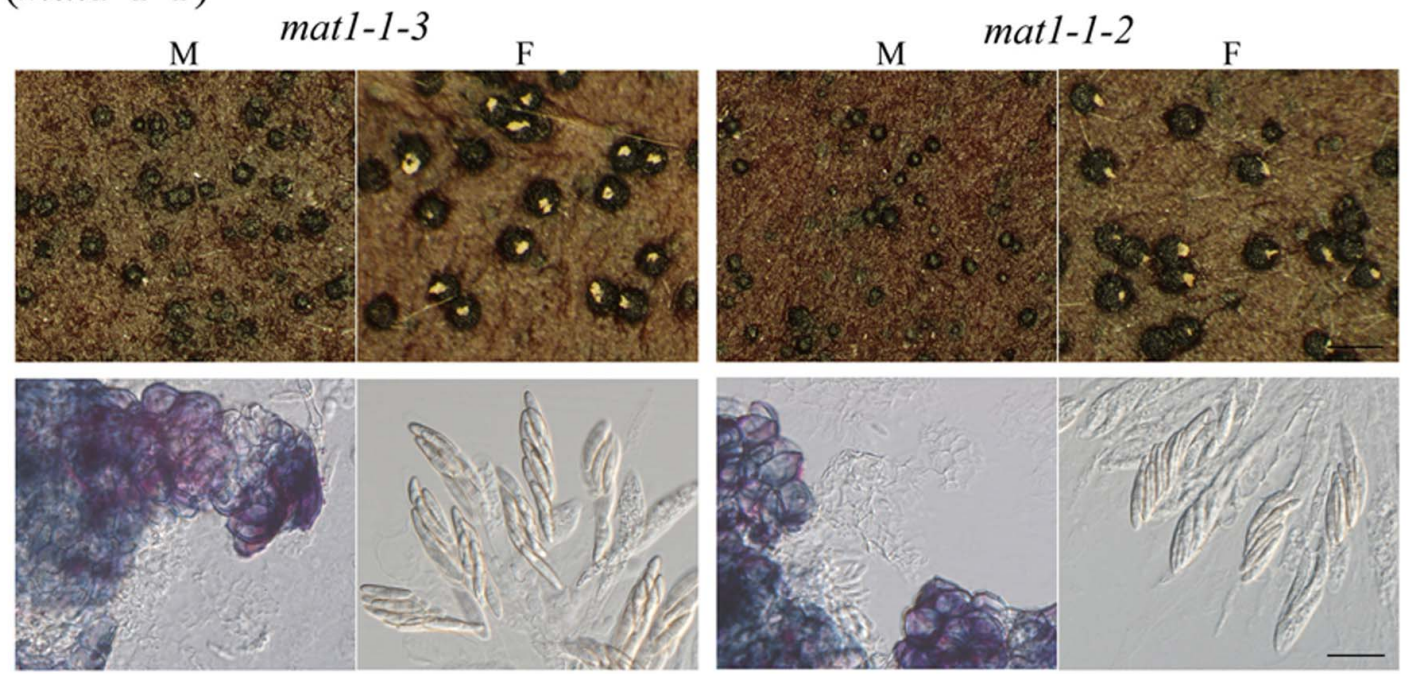

Figure 6. Outcrossing defects in the mat 1-2-1 and various mat1-1 mutants. A. The mat1-2-1 mutant was crossed as the male (M) or female (F) with the mat 1-1-3, mat1-1-2, mat1-1-1, and mat1-1 mutants. B. The mat 1-1-1 mutant was crossed as the male (M) or female (F) with the mat1-1-3 and mat 1-1-2 mutants. The upper panels showed the size of representative perithecia and production of ascospore cirrhi $(\mathrm{bar}=1 \mathrm{~mm})$. The lower panels showed perithecia with or without fascicles of asci $(\mathrm{bar}=20 \mu \mathrm{m})$. doi:10.1371/journal.pone.0066980.g006 
Table 2. Defects of different mutants in outcrossing.

\begin{tabular}{lll}
\hline Female strain $^{*}$ & Male $^{*}$ & Perithecium and ascospore formation \\
\hline mat1-1-1 mutant & mat1-1-2 & Normal \\
\hline mat1-1-2 mutant & mat1-2-1 & Normal \\
& mat1-1-1 & Partially normal perithecia, defective in ascospore releasing \\
& mat1-1-3 & Small, sterile perithecia \\
\hline mat1-1-3 mutant & mat1-2-1 & Normal \\
\hline mat1-1-1 & mat1-1-2 & Normal \\
& mat1-2-1 & Small, sterile perithecia \\
\hline mat1-1-1 & Normal \\
\hline mat1-1-2 & Normal \\
\hline & mat1-1-3 & Small, sterile perithecia \\
\hline & mat1-1 & Rare \\
\hline
\end{tabular}

*Carrot agar (CA) cultures fertilized with conidia harvested from CMC cultures. Perithecium development and ascospore formation were examined 10 days postperithecial induction.

doi:10.1371/journal.pone.0066980.t002

A
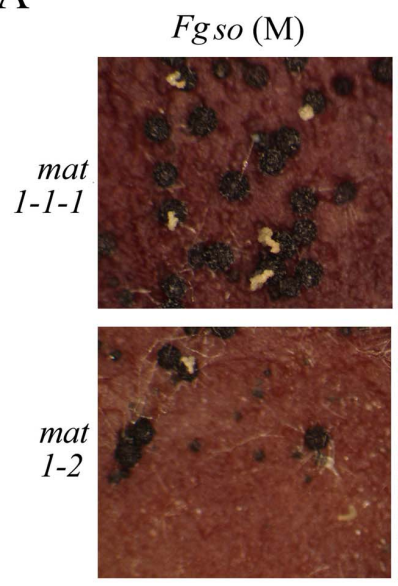

B

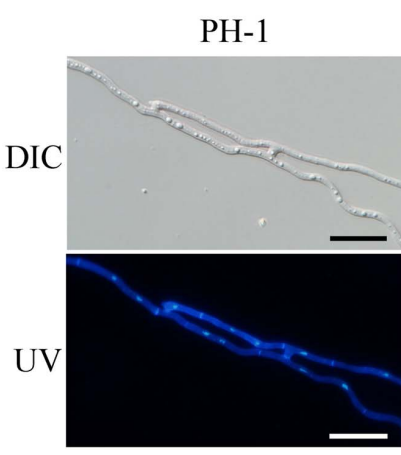

Fgso $(\mathrm{F})$
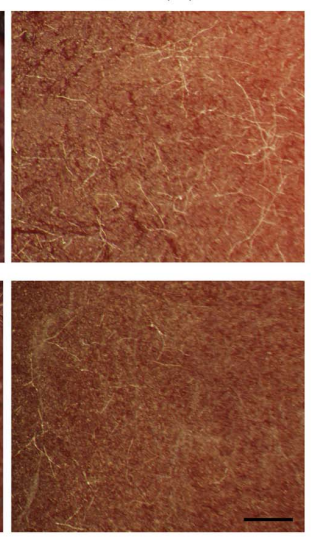

mat1-1-1

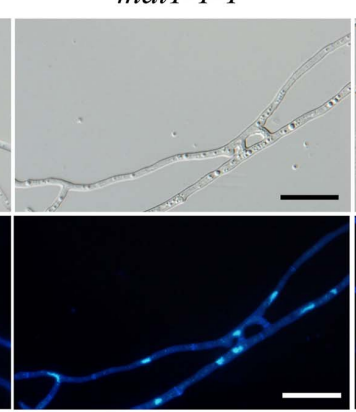

C

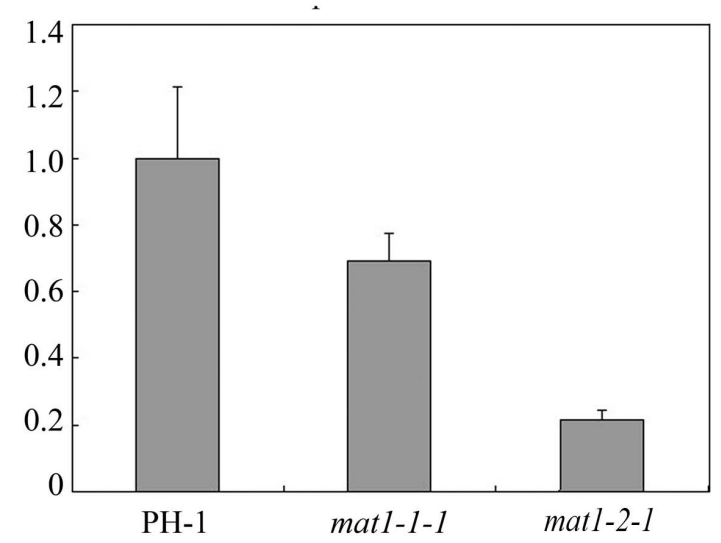

matl-2-1

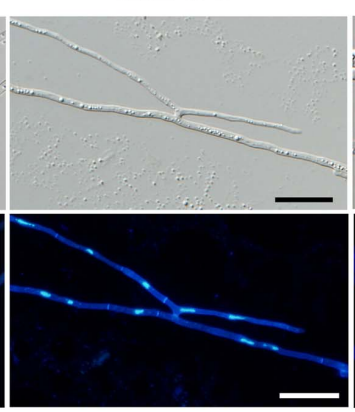

Fgso

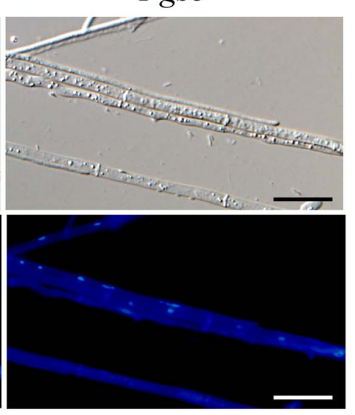

Figure 7. Mating defects of the Fgso mutant. A. The Fgso mutant was crossed as the male (M) or female (F) with the mat1-1-1 and mat1-2-1 mutants. Although it was female sterile, the Fgso mutant retained male fertility. Bar $=1 \mathrm{~mm}$. B. Assays for hyphal fusion in carrot agar cultures of the wild type (PH-1) and the mat1-1-1, mat1-2-1, and Fgso mutants. C. The expression level of FgSO in the wild type and the mat1-1-1 and mat1-2-1 mutants.

doi:10.1371/journal.pone.0066980.g007 


\section{Deletion of one MAT Gene Affects the Expression of Other MAT Locus Genes}

One possible explanation for similar phenotypes of the MAT locus gene deletion mutants is that deletion of any one of them may affect the expression of the others. To test his hypothesis, we isolated RNA from different mutants and assayed for the expression levels of individual MAT locus TF genes by qRTPCR (Fig. 8). MAT1-1-1 expression was significantly reduced in all the MAT locus gene deletion mutants assayed in comparison with the wild type, which may be related to similar mating defects in self-crosses of these mutants because MAT1-1-1 is important for male fertility. The expression of MAT1-1-2 and MAT1-1-3 was up-regulated in the mat1-1-3 and mat1-1-2 mutants, respectively (Fig. 8). Thus, we concluded that deletion of one of these two genes increased the transcription of the other, which may be related to the fact that they share the same bidirectional promoter. Both MAT1-1-2 and MAT1-1-3 were down-regulated in the mat1-1-1 and mat1-2-1 mutants (Fig. 8), indicating that their expression may be positively regulated by MAT1-1-1 and MAT1-2-1, two MAT locus genes with up-regulated expression during earlier stages of sexual reproduction (Fig. 1B). Because their close proximity, it is possible that deletion of individual MAT locus genes affect the expression of nearby genes by altering chromatin structures or promoter activities. The other possibility is that lack of one mating type protein may affect the expression of other MAT genes because they may interact with each other to form a protein complex in $F$. graminearum.

\section{Increased Expression of Pheromone or Pheromone Receptor Genes in the MAT Locus Gene Mutants}

PRE1, PRE2, PPG1, and $P P G 2$ are the pheromone and pheromone receptor genes in $F$. graminearum [29,30]. When assayed by qRT-PCR with RNA isolated from hyphae grown on 7-day-old carrot agar cultures, all the $P R E$ and $P P G$ genes were up-regulated in individual $M A T$ locus gene deletion mutants (Fig. 9). In the mat1-1-1 mutant, the expression level of PRE1, PRE2, PPG1, and PPG2 was increased 7-, 9-, 10-, and 14-fold, respectively. The up-regulation of these pheromone and pheromone receptor genes varied between 1.7- and 3.8-fold in the other MAT locus gene deletion mutants (Fig. 9). These results indicate that MAT1-1-1, and possibly other MAT locus genes, negatively regulate the expression of the PRE1, PRE2, PPG1, and PPG2 genes during vegetative growth in $F$. graminearum.

\section{Constitutive Expression of MAT1-2-1 Affects Sexual Reproduction}

The expression of MAT locus genes are repressed in vegetative hyphae in $F$. graminearum. To determine the effects of improper expression of MAT1-2-1 and MAT1-1-3, we used the constitutive TrpC promoter [31] to express these two MAT locus TF genes. The $\mathrm{P}_{\text {TrpC }}-$ MAT1-2-1 and $\mathrm{P}_{\text {TrpC }}-$ MAT1-1-3 constructs were
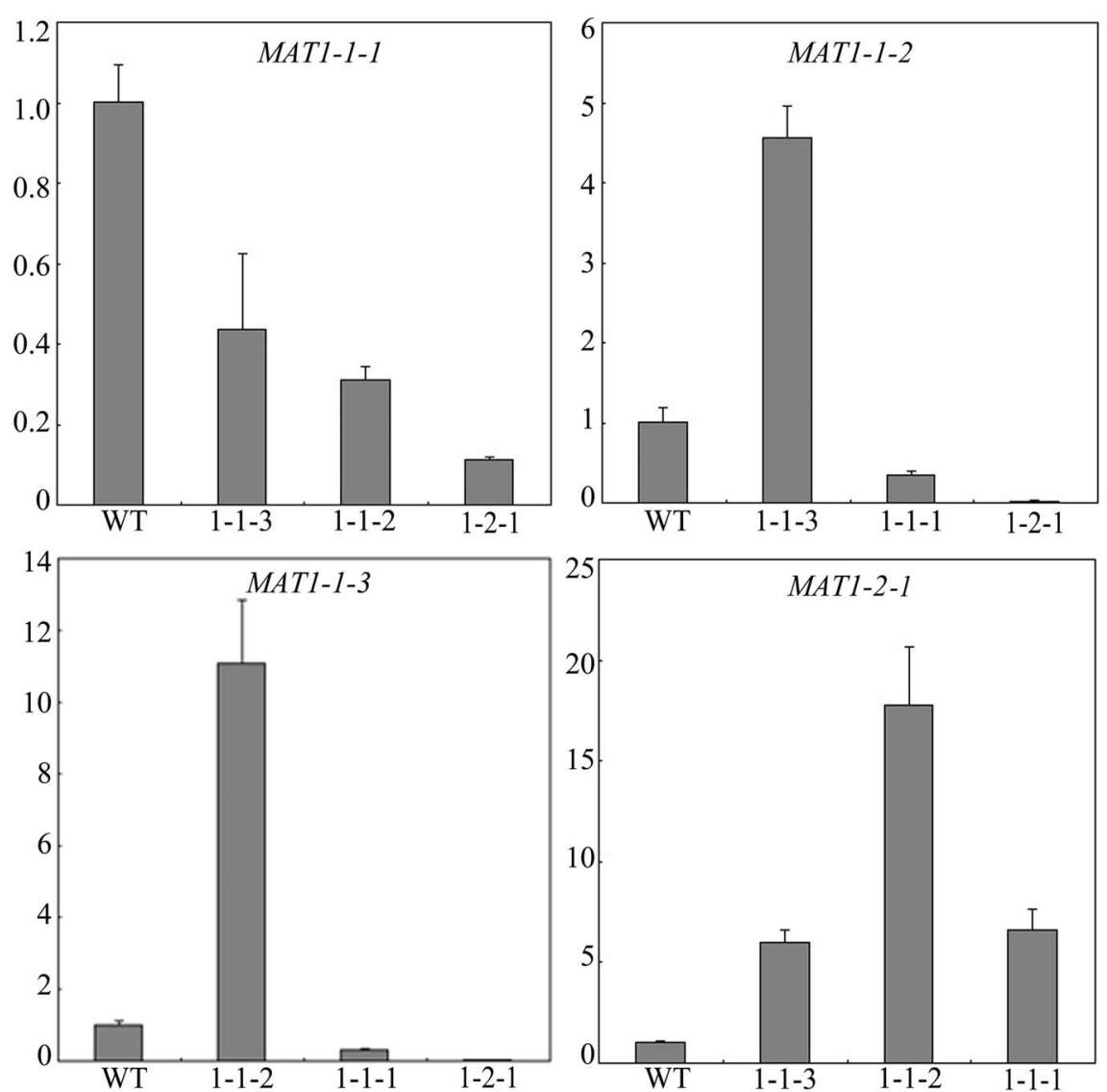

Figure 8. The expression of other MAT locus genes in the mat1-2-1 and various mat1-1 mutants. The expression of MAT1-1-1, MAT1-1-2, MAT1-1-3, and MAT1-2-1 in PH-1 (WT) or the mat1-1-1, mat1-1-2, mat1-1-3, and mat1-2-1 deletion mutants. The relative expression level of each gene in the wild type was arbitrarily set to 1. RNA samples were isolated from aerial hyphae harvested from 7-day-old carrot agar cultures. Mean and standard error were calculated from three independent biological replicates. doi:10.1371/journal.pone.0066980.g008 
transformed into $\mathrm{PH}-1$. The resulting transformants were normal in vegetative growth and asexual reproduction although GFP signals were observed in vegetative hyphae (Fig. 2B) and conidia (Fig. S6). Whereas the $\mathrm{P}_{\mathrm{TrpC}^{-}}-$MAT1-1-3 transformant had no obvious defects in sexual reproduction, the $\mathrm{P}_{\text {TrpC }}-$ MAT1-2-1 transformant produced perithecia without ascospores (Fig. 10), indicating that constitutive expression of MAT1-2-1 negatively impacted ascospore formation in F. graminearum.

\section{Discussion}

In F. graminearum, the MAT1-1-3 and MAT1-1-2 genes are likely under the control of the 259-bp bidirectional promoter and they had a similar expression profile during sexual reproduction (Fig. 1B). In $\mathcal{N}$. crassa, the mata-1 gene encodes a protein with the core binding site of CTTTG [24]. The palindromic GAAAGCTTTC sequence in this bidirectional promoter region has two CTTTG-like sequences in different strands. MAT1-2-1, a homolog of mata-1, may have similar recognition site in $F$. graminearum and bind to this palindromic sequence to regulate the expression of MAT1-1-3 and MAT1-1-2. Other transcription factors, such as $M c m 1$, may also bind to this bidirectional promoter region to negatively regulate the expression of MAT1-1-3 and MAT1-1-2 during vegetative growth or positively regulate their expression during sexual reproduction. The 259-bp promoter region has two sequences similar to the consensus Mcm1-binding site CGNNNWWRGG (rulai.cshl.edu/cgi-bin/SCPD/getfactor? MCM1).

During the preparation of this manuscript, a paper on functional characterization of the expression and knockout mutants of individual MAT locus genes in $F$. graminearum was published [9]. In this study, fungal biomass used for RNA isolation were harvested by directly scraping off hyphae and fruiting bodies together from the surface of mating cultures at 1, 4, 7, and 10 days post-perithecial induction (dpi). We found that the expression of MAT1-1-1 and MAT1-2-1 peaked at 1 dpi but MAT1-1-2 and MAT1-1-3 had the highest expression level at 4 dpi. Kim and colleague conducted qRT-PCR analysis with RNA isolated from mating cultures at 2, 4, 6, 8, 10, and 12 dpi [9]. Although there were slight differences in the expression levels of individual MAT genes among different strains of $F$. graminearum, they found that the

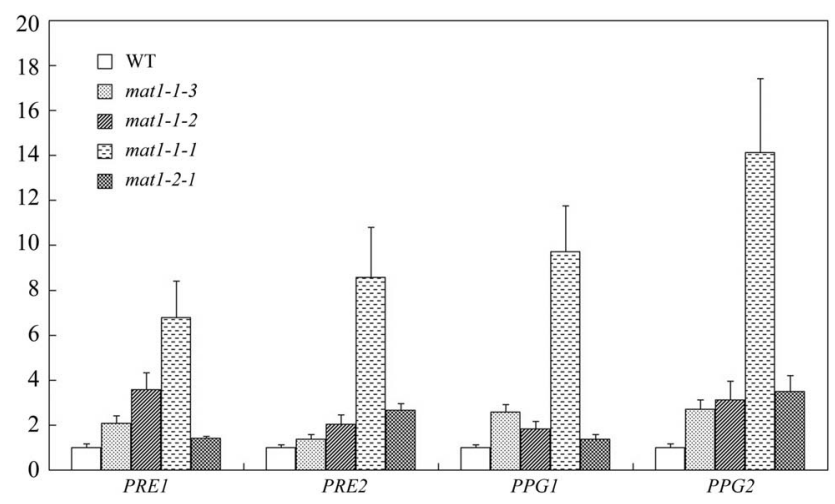

Figure 9. Effects of $M A T$ locus gene deletion on the expression of pheromone and pheromone receptor genes. The expression levels of PRE1, PRE2, PPG1, and PPG2 in PH-1 or the mat1-1-3, mat1-1-2, mat 1-1-1, and mat 1-2-1 deletion mutants were assayed by qRT-PCR. The relative expression level of each gene in the wild type was arbitrarily set to 1. RNA samples were isolated from aerial hyphae harvested from 7day-old carrot agar cultures. Mean and standard error were calculated from three independent biological replicates.

doi:10.1371/journal.pone.0066980.g009

\section{$\mathbf{A}(\mathrm{OE} 90)$}
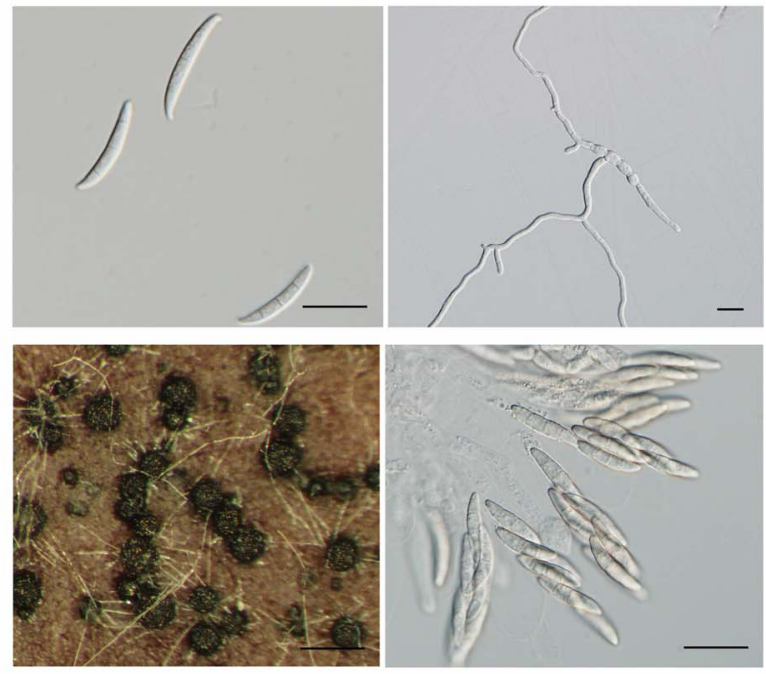

\section{B (OE93)}
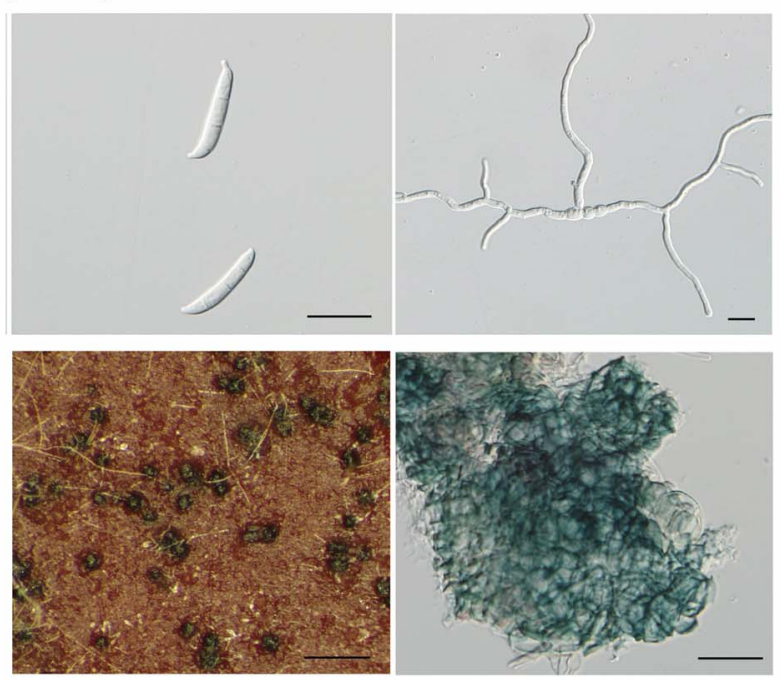

Figure 10. Vegetative growth and sexual reproduction in the $\mathbf{P}_{\text {TrpC }}$ MAT1-1-3 and $\mathrm{P}_{\text {TrpC-MAT1-2-1 transformants. A. Conidia and }}$ germ tubes of the $\mathrm{P}_{\text {TrpC }}-M A T 1-1-3$ (OE90) and $\mathrm{P}_{\text {Trpc }}-$ MAT1-2-1 (OE93) transformants. Bar $=20 \mu \mathrm{m}$. B. Carrot agar cultures $(\mathrm{bar}=1 \mathrm{~mm})$ and cracked perithecia $(\mathrm{bar}=20 \mu \mathrm{m})$ of transformants OE90 and OE93. Sterile perithecia formed by the $\mathrm{P}_{\mathrm{TrpC}}-M A T 1-2-1$ transformant were smaller than those of the $\mathrm{P}_{\mathrm{TrpC}}-M A T 1-1-3$ transformant. doi:10.1371/journal.pone.0066980.g010

expression of MAT1-1-1 and MAT1-2-1 peaked at 2 dpi and MAT1-1-2 and MAT1-1-3 had the highest expression level at 4 dpi in $\mathrm{PH} 1$ [9], which is similar to the overall expression profiles of these four MAT TFs reported in this study. However, the exact fold changes for individual MAT TF genes were different between what was reported recently [9] and our observations, which may be related to different methods used to harvest fungal biomass for RNA isolation. We noticed that Kim and colleagues separated perithecia from hyphae [9]. Because perithecium development is not synchronous, our approach may have perithecia of different development stages mixed with undifferentiated hyphae. In addition, we used $0.1 \%$ Tween 20 instead of $2.5 \%$ Tween 60 to 
induce sexual reproduction by pressing down aerial hyphae (Kim et al., 2012).

In this study, the expression levels of PPG1, PPG2, PRE1, and PRE2 in mutants deleted of individual MAT TF genes were assayed with RNA samples isolated from 7-day-old carrot agar cultures before perithecial induction. We found that the expression of all the $P R E$ and $P P G$ genes was significantly up-regulated in the mat1-1-1 mutant (Fig. 9). Their expression levels also were increased in the other MAT TF deletion mutants but not as significantly as in the mat1-1-1 mutant (Fig. 9). These results suggested that deletion of individual MAT TF genes may stimulate the expression of $P P G$ and $P R E$ genes in vegetative hyphae of $F$. graminearum. In $F$. graminearum, it has been reported that the $P P G$ and PRE genes are not essential for sexual reproduction [30]. Whereas the expression of $P P G 2$ increased at different stages of perithecial development in the mat1-1deletion mutant, up-regulation of PPG1, PRE1, and PRE2 was not observed in the mat1-1 and mat1-2 deletion mutants. Whereas Kim and colleagues [30] assayed the expression levels of the $P P G$ and $P R E$ genes in cultures after sexual induction, we assayed their expression in vegetative hyphae. Also, we used mutants deleted of individual MAT TF genes instead of the mat1-1 and mat1-2 locus deletion mutants.

One of our original goals was to determine the expression and subcellular localization of these four MAT locus genes during the switching from vegetative growth to sexual reproduction. Therefore, we generated knock-in GFP-fusion transformants in this study. Unfortunately, GFP signals were too faint or not detectable in the knock-in GFP fusion transformants of these MAT locus genes. The MAT1-1-3 knock-in transformant had the strongest GFP signals but we failed to detect fluorescence signals in the nucleus during sexual reproduction. One likely explanation is that their expression at the protein level was relatively low and perithecium tissues had a strong fluorescence background. It is also possible that MAT locus proteins only localize to the nucleus transiently. Interestingly, GFP signals were observed in the nucleus in vegetative hyphae and conidia but not in perithecium tissues of the $\mathrm{P}_{\text {TrpC }}$-MAT1-2-1-GFP transformant. In the $\mathrm{P}_{\text {TrpC }}-M A T 1-1-3-$ GFP, GFP signals in vegetative hyphae were stronger than the GFP knock-in transformant but were not localized to the nucleus (Fig. 2B).

Similar to what were reported [9], the mat1-1-1, mat1-1-2, and mat1-1-3 mutants produced smaller perithecia with thicker perithecium walls than the wild type but did not form asci or ascospores in self-crosses, indicating that all these four genes in the MAT loci are required for ascospore production. Unlike the mat12-1 mutant of GZ3639 [9], although it was reduced in perithecium formation, the mat1-2-1 mutant generated in this study still produced numerous small, sterile perithecia. Close examination revealed that ascogenous hyphal growth inside perithecia was blocked or defective in the mat1-2-1 and other MAT locus gene deletion mutants (Fig. 3).

In out-crosses, we found that the mat1-1-1 mutant displayed male-specific defects in mating although it was normal in female fertility. Kim and colleagues did not assay the defects of these MAT locus gene mutants in outcrosses [9]. To our knowledge, no other genes are known to be essential for male fertility in $F$. graminearum. In $M$. oryzae, the $m c m 1$ mutant was reported to be male sterile and it was blocked in the production of microconidia [32]. F. graminearum, unlike many other Fusarium species, does not produce microconidia. It will be important to determine the role of MAT11-1 in male fertility.

Interestingly, the mat1-2-1 deletion mutant displayed a femalespecific defect in the production of normal perithecia and ascospores in out-crosses. Most of the perithecia produced in crosses with the mat1-2-1 mutant as the female were small and sterile. Although a few of them were fertile, they failed to produce cirrhi. Therefore, MAT1-2-1 is not essential but plays an important role in perithecium development, likely before the formation of dikaryotic hyphae or diploid cells. There are many mutants known to be defective in female fertility in $F$. graminearum. Most of them are blocked in the production of proto-perithecia, including the mgv1 and Gpmk1 mutants [33,34,35,36]. The MAT11-2 and MAT1-1-3 genes were dispensable for mating either as the male or female in out-crosses. It is possible that MAT1-1-2 and MAT1-1-3 are functionally redundant. It will be of interest to generate the mat1-1-2 mat1-1-3 double mutant and determine its defects in outcrosses with other strains.

In yeast, MATa interacts with MAT $\alpha$ in diploid cells to suppress the expression of haploid-specific genes [14,25]. In F. graminearum, the dikaryotic and diploid stages are transient and occur only inside perithecia. In yeast two-hybrid assays, we observed the interaction of MAT1-1-2 with all other MAT locus genes. The interactions of MAT1-1-3 with MAT1-1-1 and MAT1-2-1 also were detected. To detect the interaction of different MAT locus genes by BiFC assays, we generated the transformants expressing different YFPG- and YFPN-fusion constructs (Table 1). Unfortunately, we failed to observe YFP signals in any of these transformants during vegetative growth or sexual reproduction. The expression levels of these MAT locus genes may be too low and fluorescence background may be too high in perithecium tissues. It is also possible that the interactions among the MAT locus $\mathrm{TF}$ genes may be too transient to be detected by BiFC assays.

In $\mathcal{N}$. crassa, SO is important for hyphal fusion. The so mutant displayed pleiotropic defects in growth, conidiation, and sexual reproduction $[27,28]$. Orthologs of $S O$ are well conserved in filamentous ascomycetes but it is absent in yeast and its function in plant pathogenic fungi is not clear. In F. graminearum, the Fgso mutant was defective in hyphal fusion and sterile in self-crosses. It was female sterile but male fertile in outcrosses. In infection assays, the Fgsol mutant was significantly reduced in virulence. Because $\mathrm{FgSO}$ expression and hyphal fusion were reduced in the mat1-2-1 mutant, it is possible that MAT1-2-1 may directly or indirectly regulate the expression of $\mathrm{FgSO}$ in $\mathrm{F}$. graminearum.

Interestingly, although the MAT locus gene deletion mutants were normal in wheat head infection, we noticed that the mat1-1-1 and mat1-2-1 mutants, but not the mat1-1-2 and mat1-1-3 mutants, were reduced in virulence in corn stalk rot assays. The same results were obtained in repeated experiments. The mat1-1-1 and mat1-21 mutants may be defective in adaptation to or colonization of corn stalks, which are substrates for $F$. graminearum to produce perithecia in the field. It will be important to further characterize the defects of the mat1-1-1 and mat1-2-1 mutants in corn stalk infection and determine the underlying mechanism. Corn stalks are known to accumulate diterpenoid phytoalexins [37]. The mat11-1 and mat1-2-1 mutants may have enhanced sensitivity to these phytoalexins or may be defective in overcoming other plant defensive responses in corn stalks.

\section{Materials and Methods}

\section{Strains and Culture Conditions}

The wild-type strain and mutants of $F$. graminearum used in this study are listed in Table 1 . Cultures were routinely grown on PDA plates at $25^{\circ} \mathrm{C}[38,39]$. Conidiation in 5-day-old $\mathrm{CMC}$ cultures and growth rate on PDA plates were measured as described $[40,41]$. For DNA extraction, vegetative hyphae were harvested from 2 -day-old YEPD (1\% yeast extract, $2 \%$ peptone, $2 \%$ glucose) 
cultures. For genetic crosses, aerial hyphae of 7-day-old carrot agar cultures were pressed down with $300 \mu \mathrm{l}$ of sterile $0.1 \%$ Tween 20 or conidium suspensions $\left(10^{5}\right.$ conidia/ml $)$ of the male strains and incubated under black light [42]. Perithecium formation and cirrhi production were assayed after incubation at $25^{\circ} \mathrm{C}$ for 2 weeks. Protoplast preparation and fungal transformation were performed as described $[33,43,44]$. For transformation, hygromycin B (Calbiochem, La Jolla, CA) and geneticin (SigmaAldrich, St. Louis, MO) were added to the final concentration of 250 and $150 \mu \mathrm{g} / \mathrm{ml}$, respectively [45].

\section{Plant Infection Assays}

For wheat head and corn stalk infection assays, freshly harvested conidia were re-suspended to $10^{5} \mathrm{spores} / \mathrm{ml}$ in sterile distilled water. Flowering wheat heads of cultivar Xiaoyan22 were inoculated with $10 \mu \mathrm{l}$ of conidium suspensions at the fifth spikelet from the base of the spike and scored for head blight symptoms as described $[46,47]$. Stalks of 8-week-old corn plants of cv. Pioneer 2375 were inoculated with toothpicks dipped in conidium suspensions as described [41,48]. Stalk rot symptoms were examined after splitting the stalks longitudinally along the inoculation site 14 dpi.

\section{qRT-PCR Analysis}

RNA samples were isolated from conidia, vegetative hyphae, and fungal biomass (hyphae and fruiting bodies) harvested from carrot agar cultures by scraping gently with a spatula with the TRIzol reagent (Invitrogen, Carlsbad, CA). For each experiment, at least three independent biological replicates were conducted. First-strand cDNA was synthesized with the Fermentas 1st cDNA synthesis kit (Hanover, MD) following the instructions provided by the manufacturer. For the internal control, the FgTUB2 betatubulin gene of $F$. graminearum was amplified with primers TubQF and TubQR [47].

\section{Generation of the mat1-1-1, mat1-1-2, mat1-1-3, mat1-2- 1, and Fgso Mutants}

The split-marker approach was used to generate the gene replacement constructs for the $M A T$ locus and $F g S O$ genes (Fig. S3; Fig. S5). Putative knockout mutants were identified by PCR and confirmed by Southern blot hybridizations to confirm the gene replacement event. All of the mutants generated in this study were preserved in $15 \%$ glycerol at $-80^{\circ} \mathrm{C}$.

\section{Generation of GFP knock-in Transformants and GFP Fusion Constructs}

A modified split-marker approach was used to generate the GFP knock-in transformants (Fig. S1). Primers $1 F / 2$ R and $3 F / 4 R$ were used to amplify the flanking sequences of the stop codons of the target genes (Fig. S1). The GFP and geneticin resistance marker $\left(\right.$ Gen $\left.^{\mathrm{R}}\right)$ fusion construct pGTP was generated in this study by overlapping PCR and used as the split marker for transformation. The resulting G418-resistant transformants were screened by PCR. In-frame fusions of GFP with MAT locus genes were then confirmed by sequencing analysis with PGR products.

To generate the MAT1-1-3-GFP and MAT1-2-1-GFP fusion constructs, PCR products containing the genomic fragments of the target genes were amplified and cloned into pFL2 [49] by the yeast gap repair approach [50]. All GFP fusion constructs were verified by sequencing analysis and transformed into protoplasts of the corresponding mutants. G418-resistant transformants harboring the transforming constructs were identified by PCR and confirmed by the presence of GFP signals.

\section{Yeast Two-hybrid Assays}

Protein-protein interactions were assayed with the Matchmaker yeast two-hybrid system (Clontech, Mountain View, CA). ORFs of the MAT1-1-2, MAT1-1-3, MAT1-2-1, and MCM1 were amplified from first-strand cDNA of $\mathrm{PH}-1$ and cloned into pGBK7 (Clontech) as the bait constructs. For the MAT1-1-1, MAT1-2-1, and MCM1 genes, their ORFs were amplified and cloned into pGADT7 as the prey constructs. The resulting bait and prey vectors were co-transformed in pairs into yeast strain AH109 (Clontech). The Leu+ and Trp+ transformants were isolated and assayed for growth on SD-Trp-Leu-His medium and galactosidase activities with filter lift assays as described [51]. The positive and negative controls were provided in the Matchmaker library construction kit (Clontech).

\section{BiFC Assays}

Plasmids pHZ65 and pHZ68 containing the N-terminal (1154aa) and C-terminal (155-238 aa) regions of YFP [52], respectively, were used to generate the YFPN- or YFPC-fusion constructs. The MAT1-1-1-YFPN fusion construct was generated by cloning the MAT1-1-1 gene amplified with primers M1native and M1YFP into pHZ65 by yeast gap repair [50]. A similar approach was used to generate the YFPN- or YFPC-fusion constructs of MAT1-1-2, MAT1-1-3, and MAT1-2-1. Pairs of the resulting BiFG vectors were then transformed into protoplasts of $\mathrm{PH}-1$. The resulting transformants (Table 1) were screened by PCR for the presence of the target YFPN- or YFPC-fusion constructs.

\section{Supporting Information}

Figure S1 Generation of in-frame MAT1-2-1-GFP knockin fusion transformants. A. Diagram for the MAT1-2-1 knock-in construct. The GFP-G418 resistant marker fragment from pGTP was used to replace the terminator sequence of MAT1-2-1. The lower panel showed PCR verification of GFP knock-in transformants (1-14). M, marker; WT, wild type. B. GFP signals in the MAT1-2-1-GFP knock-in transformant in conidia and hyphae. Bar $=20 \mu \mathrm{m}$.

(TIF)

Figure S2 Generation of the gene replacement mutants of four MAT locus TF genes. A. TheMAT1-1-1 locus and gene replacement construct. TheMAT1-1-1 and $h p h$ genes are marked with empty and black arrows, respectively. $1 \mathrm{~F}, 2 \mathrm{R}, 3 \mathrm{~F}$, and $4 \mathrm{R}$ are the primers used to amplify the flanking sequences. Lower panels are Southern blots of the wild type (PH-1) and putative mat1-1-1 mutants (M1, M2, and M3) hybridized with probe A (left) amplified with primers $\mathrm{M} 15 \mathrm{~F} / \mathrm{M} 16 \mathrm{R}$ and probe $\mathrm{B}$ (right) amplified with primers H852/H850. Panels B, G, and D were similar figures showing the gene replacement constructs and mutants of the MAT1-1-2, MAT1-1-3, and MAT1-2-1 genes, respectively. E, EcoRI; K, $K p n \mathrm{I} ; \mathrm{X}, X b a \mathrm{I}$ (TIF)

Figure S3 Three-day-old PDA cultures of the wild type and the mat1-1-3, mat1-1-2, mat1-1-1, and mat1-2-1 mutants. No differences in growth or colony morphology were observed between PH- 1 and the mutants.

(TIF)

Figure S4 Conidia and $12 \mathrm{~h}$ germ tubes of the wild type (PH-1) and the mat1-1-3, mat1-1-2, mat1-1-1, and mat1-2-1 mutants. Bar $=20 \mu \mathrm{m}$.

(TIF) 
Figure S5 Generation of the Fgso deletion mutant. A. The FgSO gene replacement construct (upper panel) and verification of the Fgso deletion mutants by Southern blot analysis (lower panel). Genomic DNA samples were digested with EcoRV (E). WT, the wild type strain PH-1. M1-M4, putative Fgso mutants. B. Three-day-old PDA cultures of PH-1 and the Fgso deletion mutant.

(TIF)

Figure $S 6$ GFP signals in the conidia of $P_{\text {TrpC }}-M A T 1-2-1$ GFP transformant. The localization of GFP signals in the nucleus and cytoplasm of conidia harvested from 5-day-old CMC cultures of the $\mathrm{P}_{\text {TrpC }}$-MAT1-2-1-GFP transformant.

(TIF)

\section{References}

1. Goswami RS, Kistler HC (2004) Heading for disaster: Fusarium graminearum on cereal crops. Mol Plant Pathol 5: 515-525.

2. Bai GH, Shaner G (2004) Management and resistance in wheat and barley to Fusarium head blight. Annu Rev Phytopathol 42: 135-161.

3. Trail F (2009) For blighted waves of grain: Fusarium graminearum in the postgenomics era. Plant Physiol 149: 103-110.

4. Desjardins AE (2003) Gibberella from A (venaceae) to Z (eae). Annu Rev Phytopathol 41: 177-198.

5. Yun SH, Arie T, Kaneko I, Yoder OC, Turgeon BG (2000) Molecular organization of mating type loci in heterothallic, homothallic, and asexual Gibberella/ Fusarium species. Fungal Genet Biol 31: 7-20.

6. Lee J, Lee T, Lee YW, Yun SH, Turgeon BG (2003) Shifting fungal reproductive mode by manipulation of mating type genes: obligatory heterothallism of Gibberella zeae. Mol Microbiol 50: 145-152.

7. Martin T, Lu SW, van Tilbeurgh H, Ripoll DR, Dixelius C, et al. (2010) Tracing the origin of the fungal alpha 1 domain places its ancestor in the HMGbox superfamily: implication for fungal mating-type evolution. PLoS One 5

8. Martin SH, Wingfield BD, Wingfield MJ, Steenkamp ET (2011) Structure and evolution of the Fusarium mating type locus: New insights from the Gibberella fuïkuroi complex. Fungal Genet Biol 48: 731-740.

9. Kim HK, Cho EJ, Lee S, Lee YS, Yun SH (2012) Functional analyses of individual mating-type transcripts at MAT loci in Fusarium graminearum and Fusarium asiaticum. FEMS Microbiol Lett 337: 89-96.

10. Klix V, Nowrousian M, Ringelberg C, Loros JJ, Dunlap JC, et al. (2010) Functional characterization of MAT1-1-specific mating-type genes in the homothallic ascomycete Sordaria macrospora provides new insights into essential and nonessential sexual regulators. Eukaryot Cell 9: 894-905.

11. Debuchy R, Berteaux-Lecellier V, Silar P (2010) Mating systems and sexual morphogenesis in ascomycetes. In: Borkovich KA, Ebbole DJ, editors. Cellular and Molecular Biology of Filamentous Fungi. 501-535.

12. Ferreira AVB, Saupe S, Glass NL (1996) Transcriptional analysis of the $m t A$ idiomorph of Neurospora crassa identifies two genes in addition to $m t A-1$. Mol Gen Genet 250: 767-774.

13. Lee SC, Ni M, Li W, Shertz C, Heitman J (2010) The evolution of sex: a perspective from the fungal kingdom. Microbiol Mol Biol Rev 74: 298-325.

14. Haber JE (2012) Mating-type genes and MAT switching in Saccharomyces cerevisiae. Genetics 191: 33-64

15. Tuch BB, Galgoczy DJ, Hernday AD, Li H, Johnson AD (2008) The evolution of combinatorial gene regulation in fungi. PLoS Biol 6: 352-364.

16. Tsong AE, Tuch BB, Johnson AD (2007) Rewiring transcriptional circuitry: Mating-type regulation in Saccharomyces cerevisiae and Candida albicans as a model for evolution. In: Heitman J, Kronstad JW, Taylor JW, Casselton LA, editors. Sex in Fungi: Molecular Determination and Evolutionary Implications. 75-89.

17. Morohashi N, Yamamoto Y, Kuwana S, Morita W, Shindo H, et al. (2006) Effect of sequence-directed nucleosorne disruption on cell-type-specific repression by alpha 2/Mcml in the yeast genorne. Eukaryot Cell 5: 1925-1933.

18. Badgett TC, Staben C (1999) Interaction between and transactivation by the mating type polypeptides of Neurospora crassa. Proc Fungal Genet Conf 20: 127.

19. da Silva CG, Cruz RC, Bucciarelli-Rodriguez M, Vilas-Boas A (2009) Neurospora crassa Mat A-2 and Mat A-3 proteins weakly interact in the yeast two-hybrid system and affect yeast growth. Genet Mol Biol 32: 354-361.

20. Nolting N, Poeggeler S (2006) A MADS box protein interacts with a mating-type protein and is required for fruiting body development in the homothallic ascomycete Sordaria macrospora. Eukaryot Cell 5: 1043-1056.

21. Jacobsen S, Wittig M, Poggeler S (2002) Interaction between mating-type proteins from the homothallic fungus Sordaria macrospora. Curr Genet 41: 150158.

22. Coppin E, Debuchy R, Arnaise S, Picard M (1997) Mating types and sexual development in filamentous ascomycetes. Microbiol Mol Biol Rev 61: 411-428.

23. Adachi N, Lieber MR (2002) Bidirectional gene organization: A common architectural feature of the human genome. Cell 109: 807-809.

24. Philley ML, Staben C (1994) Functional analyses of the Neurospora-crassa $m t a-1$ mating-type polypeptide. Genetics 137: 715-722.

\section{Acknowledgments}

We thank Dr. S. H. Yun at Soonchunhyang University for providing the mat1-1 deletion mutant and Dr. Larry Dunkle for critical reading of this manuscript. We also thank Dr. Guotian Li for the GFP knock-in construct and Dr. Cong Jiang for assistance with qRT-PCR analysis.

\section{Author Contributions}

Conceived and designed the experiments: JX. Performed the experiments: QZ RH JZ JM ZW GW CW. Analyzed the data: QZ RH. Contributed reagents/materials/analysis tools: QZ RH GW. Wrote the paper: QZ JX.

25. Gelli A (2002) Rst1 and Rst2 are required for the a/alpha diploid cell type in yeast. Mol Microbiol 46: 845-854.

26. Zhong HL, McCord R, Vershon AK (1999) Identification of target sites of the alpha 2-Mcml repressor complex in the yeast genome. Genome Res 9: 10401047.

27. Fleissner A, Glass NL (2007) SO, a protein involved in hyphal fusion in Neurospora crassa, localizes to septal plugs. Eukaryot Cell 6: 84-94.

28. Fleissner A, Sarkar S, Jacobson DJ, Roca MG, Read ND, et al. (2005) The so locus is required for vegetative cell fusion and postfertilization events in Neurospora crassa. Eukaryot Cell 4: 920-930.

29. Lee JK, Leslie JF, Bowden RL (2008) Expression and function of sex pheromones and receptors in the homothallic ascomycete Gibberella zeae. Eukaryot Cell 7: 1211-1221.

30. Kim HK, Lee T, Yun SH (2008) A putative pheromone signaling pathway is dispensable for self-fertility in the homothallic ascomycete Gibberella zeae. Fungal Genet Biol 45: 1188-1196.

31. Hamer JE, Timberlake WE (1987) Functional-organization of the Aspergillus nidulans Trpc promoter. Mol Cell Biol 7: 2352-2359.

32. Zhou X, Li G, Xu JR (2011) Efficient approaches for generating GFP fusion and epitope-tagging constructs in filamentous fungi. Methods Mol Biol 722: 199 212.

33. Hou ZM, Xue CY, Peng YL, Katan T, Kistler HC, et al. (2002) A mitogenactivated protein kinase gene $(M G V 1)$ in Fusarium graminearum is required for female fertility, heterokaryon formation, and plant infection. Mol Plant-Microbe Interact 15: 1119-1127.

34. Jenczmionka NJ, Schafer W (2005) The Gpmk1 MAP kinase of Fusarium graminearum regulates the induction of specific secreted enzymes. Curr Genet 47: 29-36.

35. Zheng DW, Zhang SJ, Zhou XY, Wang CF, Xiang P, et al. (2012) The FgHOG1 pathway regulates hyphal growth, stress responses, and plant infection in Fusarium graminearum. Plos One 7.

36. Urban M, Mott E, Farley T, Hammond-Kosack K (2003) The Fusarium graminearum MAP1 gene is essential for pathogenicity and development of perithecia. Mol Plant Pathol 4: 347-359.

37. Schmelz EA, Kaplan F, Huffaker A, Dafoe NJ, Vaughan MM, et al. (2011) Identity, regulation, and activity of inducible diterpenoid phytoalexins in maize. Proc Natl Acad Sci USA 108: 5455-5460.

38. Seong K, Hou ZM, Tracy M, Kistler HC, Xu JR (2005) Random insertional mutagenesis identifies genes associated with virulence in the wheat scab fungus Fusarium graminearum. Phytopathology 95: 744-750.

39. Wang Y, Liu W, Hou Z, Wang C, Zhou X, et al. (2011) A novel transcriptional factor important for pathogenesis and ascosporogenesis in Fusarium graminearum. Mol Plant-Microbe Interact 24: 118-128.

40. Ding SL, Mehrabi R, Koten C, Kang ZS, Wei YD, et al. (2009) Transducin beta-like gene FTL1 is essential for pathogenesis in Fusarium graminearum. Eukaryot Cell 8: 867-876.

41. Zhou XY, Heyer C, Choi YE, Mehrabi R, Xu JR (2010) The CID1 cyclin C-like gene is important for plant infection in Fusarium graminearum. Fungal Genet Biol 47: 143-151.

42. Li Y, Wang C, Liu W, Wang G, Kang Z, et al. (2011) The HDF1 histone deacetylase gene is important for conidiation, sexual reproduction, and pathogenesis in Fusarium graminearum. Mol Plant-Microbe Interact 24: 487-496.

43. Proctor RH, Hohn TM, McCormick SP (1995) Reduced virulence of Gibberella zeae caused by disruption of a trichothecene toxin biosynthetic gene. Mol PlantMicrobe Interact 8: 593-601.

44. Seong K, Li L, Hou ZM, Tracy M, Kistler HC, et al. (2006) Cryptic promoter activity in the coding region of the HMG-CoA rediactase gene in Fusarium graminearum. Fungal Genet Biol 43: 34-41.

45. Wang G, Wang C, Hou R, Zhou X, Li GH, et al. (2012) The AMT1 arginine methyltransferase gene is important for plant infection and normal hyphal growth in Fusarium graminearum. PLoS One 7: e38324. 
46. Gale LR, Chen LF, Hernick CA, Takamura K, Kistler HC (2002) Population analysis of Fusarium graminearum from wheat fields in eastern China. Phytopathology 92: 1315-1322.

47. Bluhm BH, Zhao X, Flaherty JE, Xu JR, Dunkle LD (2007) RAS2 regulates growth and pathogenesis in Fusarium graminearum. Mol Plant-Microbe Interact 20: 627-636.

48. Choi YE, Xu J-R (2010) The cAMP signaling pathway in Fusarium verticillioides is important for conidiation, plant infection, and stress responses but not fumonisin production. Mol Plant-Microbe Interact 23: 522-533.

49. Zhou X, Xu JR (2011) Efficient approaches for generating GFP fusion and epitope-tagging constructs in filamentous fungi. In: Xu JR, Bluhm B, editors.
Fungal Genomics: Methods and Protocols. Heidelberg: Humana Press. 199212.

50. Bruno KS, Tenjo F, Li L, Hamer JE, Xu JR (2004) Cellular localization and role of kinase activity of PMK1 in Magnaporthe grisea. Eukaryot Cell 3: 1525-1532.

51. Park G, Xue G, Zhao X, Kim Y, Orbach M, et al. (2006) Multiple upstream signals converge on an adaptor protein Mst50 to activate the PMK1 pathway in Magnaporthe grisea. Plant Cell 18: 2822-2835.

52. Zhao XH, Xu JR (2007) A highly conserved MAPK-docking site in Mst7 is essential for Pmkl activation in Magnaporthe grisea. Mol Microbiol 63: 881-894.

53. Cuomo CA, Gueldener U, et al. (2007) The Fusarium graminearum genome reveals a link between localized polymorphism and pathogen specialization. Science 317: 1400-1402. 